\title{
A CLASSIFICATION OF ISOTROPIC AFFINE HYPERSPHERES
}

\author{
MARILENA MORUZ AND LUC VRANCKEN
}

\begin{abstract}
We study affine hypersurfaces $M$ which have isotropic difference tensor. Note that any surface always has isotropic difference tensor. In case that the metric is positive definite such hypersurfaces have been previously studied in [2] and [?]. We first show that the dimension of an isotropic affine hypersurface is either $5,8,14$ or 26 . Next we assume that $M$ is an affine hypersphere and we obtain in each of the possible dimensions a complete classification.
\end{abstract}

\section{INTRODUCTION}

The notion of a submanifold with isotropic second fundamental form was first introduced in [12] by O'Neill for immersions if Riemannian manifolds and recently extended by Cabrerizo et al. in [4] for pseudo-Riemannian manifolds. We say that $M$ has isotropic second fundamental form $h$ if and only if for any tangent vector $X$ at a point $p$ we have that

$$
<h(X(p), X(p)), h(X(p), X(p))>=\lambda(p)<X(p), X(p)>^{2} .
$$

If $\lambda$ is independent of the point $p$, the submanifold is called constant isotropic. Given the similarities between the basic equations that charactherise the manifolds and the important role played by the difference tensor it is natural to introduce the equivalent notion of isotropy in affine geometry. That is, a hypersphere $M$ has isotropic difference tensor $K$ if and only if for any tangent vector $X$ at a point $p$ we have that

$$
h(K(X(p), X(p)), K(X(p), X(p)))=\lambda(p) h(X(p), X(p))^{2},
$$

where $h$ is the affine metric on the hypersurface. Note that a 2-dimensional affine surface is always isotropic. In case that the affine metric is positive definite such submanifolds have been previously studied in [2] and [?]. In [2], beside a restriction on the dimension, a complete classification was obtained in case that the affine hypersurface is an affine sphere. In [?] a complete classification was given of 5 dimensional positive definite affine hypersurfaces.

In this paper we deal with the case that the induced affine metric has arbitrary signature. We will first show that the restriction of the dimension remains valid in the indefinite case. Even though the proof remains based on the Hurwitz theorem it is essentially different from the proof in the definite case. This is because unlike in the definite case, the unit tangent bundle at a point $p$ is no longer a compact manifold. Instead of this null vectors will play an important role in the proof of the restriction of the dimension.

In the second part of the paper we will then restrict ourselves to the case that $M$ is an affine hypersphere and we will deduce that in that case the immersion also has parallel difference tensor (and is a pseudo-Riemannian symmetric space). We then look at each of the possible dimensions and determine in each case explicitly

2010 Mathematics Subject Classification. 53A15.

Key words and phrases. Affine differential geometry, Blaschke hypersurface, affine homogeneous, isotropic difference tensor. 
by elementary means the form of the difference tensor and the possible examples. Note that for this second part also a more involved Lie group approach would be possible. We show the following theorems.

Theorem 1. Let $M^{5}$ ne a 5-dimensional affine hypersphere of $\mathbb{R}^{6}$. Assume that $M$ is $\lambda$-isotropic with $\lambda \neq 0$. Then if we identify $\mathbb{R}^{6}$ with symmetric $3 \times 3$-matrices, then $M$ is congruent with the connected component of $A=\left(\begin{array}{ccc}1 & 0 & 0 \\ 0 & -1 & 0 \\ 0 & 0 & -1\end{array}\right)$, of symmetric matrices with determinant 1.

Note that in the positive definite case we had the connected component of the identity.

Theorem 2. Let $M^{8}$ ne a 8-dimensional affine hypersphere of $\mathbb{R}^{9}$. Assume that $M$ is $\lambda$-isotropic with $\lambda \neq 0$. Then if we identify

Theorem 3. Let $M^{8}$ ne a 14-dimensional affine hypersphere of $\mathbb{R}^{9}$. Assume that $M$ is $\lambda$-isotropic with $\lambda \neq 0$. Then if we identify

Theorem 4. Let $M^{8}$ ne a 26-dimensional affine hypersphere of $\mathbb{R}^{9}$. Assume that $M$ is $\lambda$-isotropic with $\lambda \neq 0$. Then if we identify

\section{Preliminaries}

Let $f: M \longrightarrow \mathbb{R}^{n+1}$ be a nondegenerate affine hypersurface immersion. Let $D$ be the covariant derivative on $\mathbb{R}^{n+1}$ and $\Omega$ the volume form given by $\Omega\left(u_{1}, \ldots, u_{n+1}\right)=$ $\operatorname{det}\left(u_{1}, \ldots, u_{n+1}\right)$, such that $\mathbb{R}^{n+1}$ is endowed with its standard equiaffine structure $(D, \Omega)$. In a general setting, an affine manifold $\left(M^{n}, \nabla\right)$ is said to be equiaffine if there exists a volume form $\omega$, i.e. a non-vanishing $n$-form, on $M$ which is parallel with respect to $\nabla$ :

$$
\begin{aligned}
\left(\nabla_{X} \omega\right)\left(X_{1} \ldots, X_{n}\right)=X\left(\omega\left(X_{1}, \ldots, X_{n}\right)\right)-\omega\left(\nabla_{X} X_{1}, \ldots, X_{n}\right)-\ldots & \\
& -\omega\left(X_{1}, \ldots, \nabla_{X} X_{n}\right) .
\end{aligned}
$$

In this case we may also say that $(\nabla, \omega)$ is an equiaffine structure on $M^{n}$. In what follows, we briefly recall the construction of an equiaffine structure on an affine hypersurface $M^{n}$ in $\mathbb{R}^{n+1}$. For more details we refer to [13].

First, let $p \in M$ and $X, Y \in T_{p} M$. If we choose an arbitrary transversal vector field $\eta$ we can decompose

$$
D_{X} Y=\nabla_{X}^{\eta} Y+h^{\eta}(X, Y) \eta
$$

It is easy to see that $\nabla^{\eta}$ is a connection on $M$ and $h^{\eta}$ is a symmetric bilinear form. Note that the fact whether this bilinear form is degenerate or not is independent of the choice of transversal vector field $\eta$. As such $M$ is called nondegenerate if and only if this bilinear form is nondegenerate. Hence, locally there exists a volume form on $M$ associated to $h^{\eta}$, given by

$$
\omega_{h^{\eta}}\left(X_{1}, \ldots, X_{n}\right)=\sqrt{\left|\operatorname{det} h^{\eta}\left(X_{i}, X_{j}\right)\right|} .
$$

Next, we want to introduce a canonical transversal vector field $\xi$. In order to make a good choice, we define $\omega_{\eta}\left(X_{1}, \ldots, X_{n}\right):=\Omega\left(X_{1}, \ldots, X_{n}, \eta\right)$, for $X_{1}, \ldots, X_{n}$ vector fields on $M^{n}$ and we ask that the volume forms $\omega_{\xi}$ and $\omega_{h \xi}$ coincide and that $\left(\nabla^{\xi}, \omega_{\xi}\right)$ is an equiaffine structure on $M^{n}$. Notice that these conditions guarantee the existence of a unique (up to sign) transversal vector field $\xi$, see [13]. It is called the affine normal vector field, or the Blaschke normal vector field. For convenience, we will denote from now on $\nabla:=\nabla^{\xi}$. 
Finally, in terms of this transversal vector field we get for $M$ the formulas of Gauss and Weingarten, respectively, as follows:

$$
\begin{aligned}
& D_{X} Y=\nabla_{X} Y+h(X, Y) \xi, \\
& D_{X} \xi=-S X,
\end{aligned}
$$

where we call $\nabla$ the induced affine connection, $h$ the affine metric, $\xi$ the affine normal field or Blaschke normal field and $S$ the affine shape operator. An affine hypersurface is called a (proper) affine sphere if $S$ is a (non zero) multiple of the identity.

Moreover, let $R$ denote the curvature tensor of $M^{n}$. Then, the following fundamental equations hold with respect to the induced affine connection:

$$
\begin{array}{ll}
\text { Gauss equation: } & R(X, Y) Z=h(Y, Z) S X-h(X, Z) S Y \text {; } \\
\text { Codazzi equation for } h: & (\nabla h)(X, Y, Z)=(\nabla h)(Y, X, Z) ; \\
\text { Codazzi equation for } S: \quad & \left(\nabla_{X} S\right) Y=\left(\nabla_{Y} S\right) X ; \\
\text { Ricci equation: } & h(S X, Y)=h(X, S Y) ;
\end{array}
$$

The Codazzi equation implies that for a proper affine sphere, the multiple of the identity is constant, in which case by applying a homothety of the ambient space, we may assume that $S=\varepsilon I$, where $\varepsilon= \pm 1$. Moreover we have that $\xi+\varepsilon f$, where $f$ denotes the position vector, is a constant vector which is called the center of the proper affine hypersphere. By applying a translation in the ambient space we may of course always assume that the center is the origin.

As $\nabla$ is not necessarily compatible with the affine metric $h$, we can consider the difference tensor $K$, a $(1,2)$-type vector field defined as:

$$
K(X, Y)=\nabla_{X} Y-\hat{\nabla}_{X} Y
$$

where $\hat{\nabla}$ is the Levi-Civita connection on $M$. By convention, one may also write $K_{X} Y$ instead of $K(X, Y)$. The classical Berwald theorem states that $K$ vanishes identically if and only if $M$ is congruent to a nondegenerate quadric.

Proposition 5. We have the following properties for $K$ :

(1) $K(X, Y)=K(Y, X)$;

(2) for any $X$ we have $Y \mapsto K_{X} Y$ is a symmetric linear map and trace $K_{X}=0$ (the apolarity condition);

(3) $h(K(X, Y), Z)=h(K(X, Z), Y)$.

It is easy to prove that $\nabla h$ is related to $K$ by:

$$
\nabla h(X, Y, Z)=-2 h(Z, K(X, Y)) .
$$

Moreover, the equations of Gauss, Ricci and Codazzi, respectively, may also be written out with respect to the Levi-Civita connection as follows:

(2)

$$
\begin{aligned}
& \left\{\begin{array}{l}
\hat{R}(X, Y) Z=\frac{1}{2}\{h(Y, Z) S X-h(X, Z) S Y+h(S Y, Z) X-h(S X, Z) Y\}-\left[K_{X}, K_{Y}\right] Z, \\
\hat{\nabla} K(X, Y, Z)-\hat{\nabla} K(Y, X, Z)=\frac{1}{2}\{h(Y, Z) S X-h(X, Z) S Y-h(S Y, Z) X+h(S X, Z) Y,\}
\end{array}\right. \\
& \left\{\begin{array}{l}
\left(\hat{\nabla}_{X} S\right) Y-\left(\hat{\nabla}_{Y} S\right) X=K(Y, S X)-K(X, S Y), \\
(\nabla h)(X, Y, Z)=(\nabla h)(Y, X, Z),
\end{array}\right. \\
& h(X, S Y)=h(S X, Y), \\
& \text { where }
\end{aligned}
$$$$
\left[K_{X}, K_{Y}\right] Z=K_{X} K_{Y} Z-K_{Y} K_{X} Z
$$

and

$$
\hat{\nabla K}(X, Y, Z)=\hat{\nabla}_{X} K(Y, Z)-K\left(\hat{\nabla}_{X} Y, Z\right)-K\left(Y, \hat{\nabla}_{X} Z\right) .
$$


We have the following Ricci identity:

(3) $\hat{\nabla}^{2} K(X, Y, Z, W)-\hat{\nabla}^{2} K(Y, X, Z, W)=$

$$
\hat{R}(X, Y) K(Z, W)-K(\hat{R}(X, Y) Z, W)-h(Z, \hat{R}(X, Y) W) .
$$

A nondegenerate hypersurface $M$ of the equiaffine space $\mathbb{R}^{n+1}$ is called locally homogeneous if for all points $p$ and $q$ of $M$, there exists a neighborhood $U_{p}$ of $p$ in $M$, and an equiaffine transformation $A$ of $\mathbb{R}^{n+1}$, i.e. $A \in \operatorname{SL}(n+1, \mathbb{R}) \ltimes \mathbb{R}^{n+1}$, such that $A(p)=q$ and $A\left(U_{p}\right) \subset M$. If $U_{p}=M$ for all $p$, then $M$ is called homogeneous. Let $G$ be the pseudogroup defined by

$$
G=\left\{A \in \mathrm{SL}(n+1, \mathbb{R}) \ltimes \mathbb{R}^{n+1} \mid \exists U, \text { open in } M: A(U) \subset M\right\},
$$

then $M$ is locally homogeneous if and only if $G$ "acts" transitively on $M$. If $M$ is homogeneous, then $G$ is a group and every element of $G$ maps the whole of $M$ into $M$. The following proposition is probably well known, however, as we did not find an explicit reference, we include a small proof.

Proposition 6. Let $M^{n}$ be a nondegenerate homogeneous affine hypersurface. Assume that $G \subset S L(n+1, \mathbb{R})$. Then $M$ is an affine sphere centered at the origin.

Proof. We denote the immersion by $f$. Let $p$ and $q$ be in $M$ and let $g$ be the affine transformation which maps $p$ to $q$. We have that

$$
\xi(g(p))=d g(\xi(p))
$$

and

$$
d g(f(p))=g(f(p))=f(q)
$$

Moreover as $M$ is homogeneous we know that the position vector can not be a tangent vector at one point (and therefore at every point). Indeed if that were the case, we would habe a tangent vector field $X$ such that $X(p)=f(p)$. This would imply that $D_{Y} X=Y$, and therefore $h(X, Y)=0$ for any vector field $Y$. This implies that the immersion $f$ would be degenerate.

Therefore we may write $\xi=\rho f+Z$, where $Z$ is a tangent vector field and $\rho$ a function. As $M$ is locally homogeneous and $g$ belongs to $\operatorname{SL}(n+1, \mathbb{R})$ it follows that $\rho$ is constant. The construction of the affine normal of [13] then implies that $M$ is an affine sphere centered at the origin.

The equivalent notion in affine geometry for isotropic submanifolds from Riemannian geometry, which was introduced by O'Neill in [12], corresponds to submanifolds for which the difference tensor $K$ is isotropic, that is, it satisfies:

$$
h(K(X, X), K(X, X))=\lambda(p) h(X, X)^{2},
$$

for some tangent vector field $X$. Here we will always deal with the case that $\lambda \neq 0$. Therefore, if necessary, by replacing $\xi$ with $-\xi$, we may assume that $\lambda$ is positive and therefore there exists a positive function $\mu$ such that $\lambda=\mu^{2}$. We will also use the following lemma from [13]:

Lemma 7. Let $F: M \rightarrow \mathbb{R}^{n+1}$ be an equiaffine immersion. If the metric on $\mathbb{R}^{n+1}$ is indefinite, then the immersion is isotropic if and only if for any tangent vectors $X_{1}, X_{2}, X_{3}, X_{4} \in T_{p} M$, we have that

(4)

$$
\begin{gathered}
h\left(K\left(X_{1}, X_{2}\right), K\left(X_{3}, X_{4}\right)\right)+h\left(K\left(X_{1}, X_{3}\right), K\left(X_{2}, X_{4}\right)\right)+h\left(K\left(X_{1}, X_{4}\right), K\left(X_{2}, X_{3}\right)\right)= \\
\lambda(p)\left\{h\left(X_{1}, X_{2}\right) h\left(X_{3}, X_{4}\right)+h\left(X_{1}, X_{3}\right) h\left(X_{2}, X_{4}\right)+h\left(X_{1}, X_{4}\right) h\left(X_{2}, X_{3}\right)\right\} .
\end{gathered}
$$


By using lemma (7) and property (3) in Proposition 5 we get that an affine submanifold $M^{n}$ in $\mathbb{R}^{n+1}$ is isotropic if and only if for any tangent vectors $X_{1}, X_{2}, X_{3}, X_{4} \in$ $T_{p} M$ we have that

$$
\begin{aligned}
K_{X_{1}} K_{X_{2}} X_{3}+K_{X_{2}} K_{X_{1}} & X_{3}+K_{X_{3}} K_{X_{1}} X_{2}= \\
\lambda(p)\left(h\left(X_{2}, X_{3}\right) X_{1}\right. & \left.+h\left(X_{1}, X_{3}\right) X_{2}+h\left(X_{1}, X_{2}\right) X_{3}\right) .
\end{aligned}
$$

Theorem 8. ([5]) Let $\left(M_{k}^{n}, h\right)$ be an $n$-dimensional simply connected pseudoRiemannian manifold with index $k$. Let $\hat{\nabla}$ denote the Levi Civita connection, $\hat{R}$ its curvature tensor and let $T M$ denote the tangent bundle of $M_{k}^{n}$. If $K$ is a TMvalued symmetric bilinear form on $M_{k}^{n}$ satisfying that

i) $h(K(X, Y), Z)$ is totally symmetric

ii) $(\hat{\nabla} K)(X, Y, Z)=\hat{\nabla}_{X} K(Y, Z)-K\left(\hat{\nabla}_{X} Y, Z\right)-K\left(Y, \hat{\nabla}_{X} Z\right)$ is totally symmetric, iii) $\hat{R}(X, Y) Z=c(h(Y, Z) X-h(X, Z) Y)+K(K(Y, Z), X)-K(K(X, Z), Y)$, then there exists an affine immersion $\phi: M_{k}^{n} \rightarrow \mathbb{R}^{n+1}$ as an affine sphere with induced difference tensor $K$ and induced affine metric $h$.

Theorem 9. ([5]) Let $\phi^{1}, \phi^{2}: M_{k}^{n} \longrightarrow \mathbb{R}^{n+1}$ be two affine immersions of an pseudo-Riemannian $n$-manifold $\left(M_{k}^{n}, h\right)$ with difference tensors $K^{1}, K^{2}$, respectively. If

$$
h\left(K^{1}(X, Y), \phi_{*}^{1} Z\right)=h\left(K^{2}(X, Y), \phi_{*}^{2} Z\right)
$$

for all tangent vectors fields $X, Y, Z \in T_{p} M_{k}^{n}$, then there exists an isometry $\phi$ of $\mathbb{R}^{n+1}$ such that $\phi^{1}=\phi \circ \phi^{2}$.

\section{Possible dimensions And Choice of Frame}

From now on we will always assume that $M_{k}^{n}$ is an affine isotropic hypersurface in $\mathbb{R}^{n+1}$. Here $n$ denotes the dimension and $k$ the index of the affine metric. In case that the metric is definite a classification was obtained already in [2], In view of this we will also assume thay $M$ is neither positive nor negative definite, i.e. $1 \leq k<n$. Also recall that because of the properties of $K$ any surface is isotropic. Therefore we will also assume that $n>2$. First, we have the following lemma:

Lemma 10. Let $M_{k}^{n}$ be an $n$-dimensional isotropic affine hypersurface and let $p \in M_{k}^{n}$. If for any null vector $v \in T_{p} M$ we have that $K(v, v)$ is a null vector such that $h(K(v, v), v)=0$, then the difference tensor $K$ vanishes.

As its proof is very similar to the proof of Lemma 3.1 in [9], we omit it here. From now on, we will assume that $\lambda \neq 0$. By Lemma 10, there exists a null vector $v_{0}$ such that $v_{0}$ and $K\left(v_{0}, v_{0}\right)$ are linearly independent and $h\left(v_{0}, K\left(v_{0}, v_{0}\right)\right) \neq 0$. Using Lemma 7, we have that for any null vector $u$

$$
h\left(K\left(v_{0}, v_{0}\right), K\left(v_{0}, u\right)\right)=\lambda h\left(v_{0}, v_{0}\right) h\left(v_{0}, u\right)=0 .
$$

As $K_{v_{0}}$ is a symmetric operator with respect to the metric $h$, we get that $K_{v_{0}} K_{v_{0}} v_{0}=$ 0 . Moreover, taking in particular $u=v_{0}$ in $(6)$, we get that $K\left(v_{0}, v_{0}\right)$ is a null vector.

We can now take a null frame such that

$$
e_{1}=v_{0}, \quad e_{2}=K_{v_{0}} v_{0} .
$$

By rescaling $v_{0}$ if necessary, we may assume that $h\left(K\left(v_{0}, v_{0}\right), v_{0}\right)=-4 \lambda^{2}$. Then we get

$$
\begin{aligned}
& h\left(e_{1}, e_{1}\right)=h\left(e_{2}, e_{2}\right)=0 \quad h\left(e_{1}, e_{2}\right)=-4 \lambda^{2} \\
& K\left(e_{1}, e_{1}\right)=e_{2}, \quad K\left(e_{1}, e_{2}\right)=K_{v_{0}} K_{v_{0}} v_{0}=0 .
\end{aligned}
$$

Using the isotropy condition in (5) for $X_{1}=X_{2}=e_{1}, X_{3}=e_{3}$ we get that

$$
K_{e_{2}} e_{2}=-8 \lambda^{3} e_{1} \text {. }
$$


From relation (7) we can see that the space $\operatorname{span}\left\{e_{1}, e_{2}\right\}$ is invariant under the operator $K_{e_{1}}$. As the operator $K_{e_{1}}$ is symmetric with respect to the metric, it follows that also the space $\operatorname{span}\left\{e_{1}, e_{2}\right\}^{\perp}$ is invariant under $K_{e_{1}}$.

Now we follow precisely the computations of [9]. We get a basis $\left\{e_{1}, e_{2}, u_{1}, \ldots, u_{r}, \omega_{1}^{1}, \omega_{2}^{1}, \ldots, \omega_{1}^{r}, \omega_{2}^{r}\right\}$, which satisfies that $\left\{u_{1}, \ldots, u_{r}, \omega_{1}^{1}, \omega_{2}^{1}, \ldots, \omega_{1}^{r}, \omega_{2}^{r}\right\}$ is an orthogonal basis of $\left\{e_{1}, e_{2}\right\}^{\perp}$ and

$$
\left\{\begin{array}{l}
h\left(e_{1}, e_{1}\right)=h\left(e_{2}, e_{2}\right)=0, \quad h\left(e_{1}, e_{2}\right)=-4 \lambda^{2}, \\
h\left(u_{i}, u_{j}\right)=\varepsilon_{i} \delta_{i j}, \quad \varepsilon_{i}= \pm 1, \quad h\left(\omega_{1}^{\alpha}, \omega_{1}^{\alpha}\right)=1, \quad h\left(\omega_{2}^{\alpha}, \omega_{2}^{\alpha}\right)=-1,
\end{array}\right.
$$

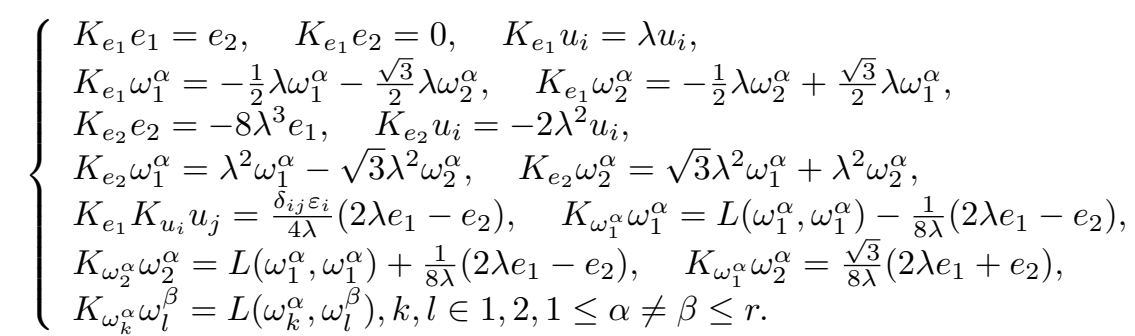

In the above formulas, $U$ and $W$ correspond to the invariant subspaces of $K_{e_{1}}$ and the operator $L$ is an operator on $W \times W$, defined by

$$
L(\omega, \tilde{\omega})=K_{\omega} \tilde{\omega}+\frac{1}{4 \lambda^{2}} h\left(K_{\omega} \tilde{\omega}, e_{2}\right) e_{1}+\frac{1}{4 \lambda^{2}} h\left(K_{\omega} \tilde{\omega}, e_{1}\right) e_{2}, \quad \omega, \tilde{\omega} \in W .
$$

which is a symmetric operator, satisfies $\operatorname{ImL} \subset U=\operatorname{span}\left\{u_{1}, \ldots, u_{k}\right\}$ and

$$
\begin{aligned}
& L\left(\omega_{1}^{\alpha}, \omega_{1}^{\alpha}\right)=L\left(\omega_{2}^{\alpha}, \omega_{2}^{\alpha}\right), \quad L\left(\omega_{1}^{\alpha}, \omega_{2}^{\alpha}\right)=0, \quad K_{\omega_{1}^{\alpha} \omega_{2}^{\alpha}}=\frac{\sqrt{3}}{4}\left(e_{1}+\frac{1}{2 \lambda} e_{2}\right), \\
& L\left(\omega_{1}^{\alpha}, \omega_{1}^{\beta}\right)=L\left(\omega_{2}^{\alpha}, \omega_{2}^{\beta}\right), \quad L\left(\omega_{1}^{\alpha}, \omega_{2}^{\beta}\right)=-L\left(\omega_{2}^{\alpha}, \omega_{1}^{\beta}\right) .
\end{aligned}
$$

As in [9] changing the frame by taking

$$
f_{1}=\left(2 \lambda e_{1}-e_{2}\right) /\left(4 \mu^{3}\right), \quad f_{2}=\left(2 \lambda e_{1}+e_{2}\right) /\left(4 \mu^{3}\right),
$$

we get that

$$
h\left(f_{1}, f_{1}\right)=-h\left(f_{2}, f_{2}\right)=\varepsilon_{0}, \quad h\left(f_{1}, f_{2}\right)=0
$$

and

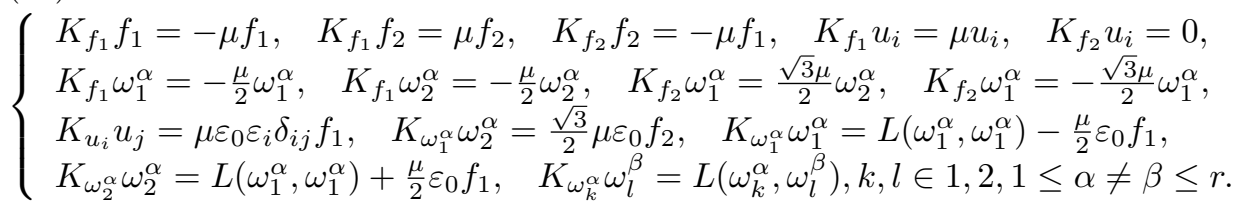

Therefore, in order to determine the difference tensor explicitly, we only need to determine all the terms $L\left(\omega_{k}^{\alpha}, \omega_{l}^{\beta}\right), k, l \in\{1,2\}, 1 \leq \alpha, \beta \leq r$. In order to do so we will summarize the above properties in a more invariant way.

Let $I$ be the identity map and define for any $w \in W$

$$
T w=\frac{2}{\sqrt{3} \lambda}\left(K_{e_{1}}+\frac{1}{2} \lambda I\right) w .
$$

We can easily check that $T$ satisfies

$$
\begin{aligned}
& T \omega_{1}^{\alpha}=\omega_{2}^{\alpha}, \quad T \omega_{2}^{\alpha}=-\omega_{1}^{\alpha}, \quad T^{2} w=-w, \quad h(T v, w)=h(v, T w), \\
& T \omega_{1}^{\alpha}=\omega_{2}^{\alpha}, \quad T \omega_{2}^{\alpha}=-\omega_{1}^{\alpha}, \quad h(T w, T v)=-h(w, v), \quad h(T v, w)=h(v, T w),
\end{aligned}
$$


for $w, v \in W$. In addition, from (11) it follows that $L(w, T v)=-L(v, T w)$ and $L(T w, T v)=L(v, w)$. We also have that $L$ satisfies an isotropy condition. Indeed, let $w=\sum_{\alpha=1}^{r} a_{\alpha} \omega_{2}^{\beta}+\sum_{\beta=1}^{r} b_{\beta} \omega_{2}^{\beta}$. By using (13) in lemma 7 we have

$$
\begin{aligned}
h\left(K_{w} w, e_{1}\right) & =\sum_{\alpha, \beta=1}^{n} a_{\alpha} a_{\beta} h\left(K_{e_{1}} \omega_{1}^{\alpha}, \omega_{1}^{\beta}\right)+\sum_{\alpha, \beta=1}^{n} b_{\alpha} b_{\beta} h\left(K_{e_{1}} \omega_{2}^{\alpha}, \omega_{2}^{\beta}\right) \\
& +\sum_{\alpha, \beta=1}^{n} a_{\alpha} b_{\beta} h\left(K_{e_{1}} \omega_{1}^{\alpha}, \omega_{2}^{\beta}\right)+\sum_{\alpha, \beta=1}^{n} b_{\alpha} a_{\beta} h\left(K_{e_{1}} \omega_{2}^{\alpha}, \omega_{1}^{\beta}\right) \\
& =-\frac{\lambda}{2} \sum_{\alpha, \beta=1}^{n}\left(a_{\alpha} a_{\beta}-b_{\alpha} b_{\beta}\right) \delta_{\alpha \beta}-\frac{\sqrt{3} \lambda}{2} \sum_{\alpha, \beta=1}^{n}\left(a_{\alpha} b_{\beta}+b_{\alpha} a_{\beta}\right) \delta_{\alpha \beta} \\
& =-\frac{\lambda}{2} h(w, w)+\frac{\sqrt{3} \lambda}{2} h(w, T w) .
\end{aligned}
$$

Similarly, we obtain

$$
h\left(K_{w} w, e_{2}\right)=\lambda^{2} h(w, w)+\sqrt{3} \lambda^{2} h(w, T w) .
$$

By combining (10), (16) and (17) we get

$$
\begin{aligned}
h(L(w, w), L(w, w)) & =h\left(K_{w} w, K_{w} w\right)+\frac{1}{2 \lambda^{2}} h\left(K_{w} w, e_{1}\right) h\left(K_{w} w, e_{2}\right) \\
& =\lambda h(w, w)^{2}+\frac{1}{2 \lambda^{2}}\left(-\frac{1}{2} \lambda^{3} h(w, w)^{2}+\frac{3}{2} \lambda^{3} h(w, T w)^{2}\right) \\
& =\frac{3}{4} \lambda\left(h(w, w)^{2}+h(w, T w)^{2}\right) .
\end{aligned}
$$

Liniarizing the previous expression for arbitrary vectors $W_{1}, W_{2}, W_{3}, W_{4} \in W$, we obtain:

$$
\begin{gathered}
h\left(L\left(W_{1}, W_{2}\right), L\left(W_{2}, W_{3}\right)\right)+h\left(L\left(W_{1}, W_{3}\right), L\left(W_{2}, W_{4}\right)\right)+h\left(L\left(W_{1}, W_{4}\right), L\left(W_{2}, W_{3}\right)\right) \\
=\frac{3}{4}\left(h\left(W_{1}, W_{2}\right) h\left(W_{3}, W_{4}\right)+h\left(W_{1}, W_{3}\right) h\left(W_{2}, W_{4}\right)+h\left(W_{1}, W_{4}\right) h\left(W_{2}, W_{3}\right)\right. \\
\left.+h\left(W_{1}, T W_{2}\right) h\left(W_{3}, T W_{4}\right)+h\left(W_{1}, T W_{3}\right) h\left(W_{2}, T W_{4}\right)+h\left(W_{1}, T W_{4}\right) h\left(W_{2}, T W_{3}\right)\right) .
\end{gathered}
$$

Note that given a metric of neutral signature on $\left\{f_{1}, f_{2}\right\}^{\perp}$ and operators $T$ and $L$ satisfying the previous conditions, we can define a frame such that (14) holds. We start with a vector $u \in\left\{f_{1}, f_{2}\right\}^{\perp}$ with length 1 . Then $T u$ has length -1 . We now write $w=a u+b T u$. The fact that $w$ has length 1 and is orthogonal to $T w$. Then implies that

$$
\begin{aligned}
& \left(a^{2}-b^{2}\right)+2 a b<u, T u>=1 \\
& \left(a^{2}-b^{2}\right)<u, T u>-2 a b=0,
\end{aligned}
$$

which determines $a$ and $b$ uniquely upto sign. It is then sufficient to take $w_{1}^{1}=w$ and $w_{2}^{1}=T w$ and to complete the construction is an inductive way.

In what follows we are going to determine the possible dimensions of the studied submanifold $M^{n}$. In order to do this, we will use a well known result from the theory of composition of quadratic forms, namely the '1,2,4,8 Theorem' proved by Hurwitz in 1898. One can find it for example in [15]. It states that there exists an $n$-square identity over the complex numbers of the form

$$
\left(x_{1}^{2}+\ldots+x_{n}^{2}\right)\left(y_{1}^{2}+\ldots+y_{n}^{2}\right)=z_{1}^{2}+\ldots+z_{n}^{2},
$$


where $X=\left(x_{1}, \ldots, x_{n}\right)$ and $Y=\left(y_{1}, \ldots, y_{n}\right)$ are systems of indeterminates and each $z_{k}=z_{k}(X, Y)$ is a bilinear form in $X$ and $Y$, if and only if $n=1,2,4$ or 8 .

We are going to see how this result applies in our case and then determine the values of $L$ on the components of the basis in order to determine the difference tensor of our immersion.

In order to apply the 1,2,4,8 Theorem, we are going to find conveniently defined complex vector spaces and an operator which preserves lengths.

First, we denote by $U^{\mathbb{C}}$ the complex linear extension of $V$ and by $W^{\mathbb{C}}$ the complex linear extension of $W$. We now take

$$
\begin{aligned}
& \mathcal{W}_{1}=\{v+i T v \mid v \in W\}, \\
& \mathcal{W}_{2}=\{w-i T w \mid w \in W\} .
\end{aligned}
$$

Note that these are indeed complex linear vector spaces as $i(v \pm i T v)=\mp(T v \mp i v)=$ $(\mp T v \pm i T(\mp T v))$ and we complexify the metric and the previously defined operator $L$. Note that $L$ is symmetric and that from the properties of $L$ and $T$ it follows that the restriction of $L$ to $\mathcal{W}_{1} \times \mathcal{W}_{1}$ and $\mathcal{W}_{2} \times \mathcal{W}_{2}$ vanishes identically. Therefore in order to determine $L$ it is sufficient to study $L$ on

$$
\left\{\begin{array}{l}
L: \mathcal{W}_{1} \times \mathcal{W}_{2} \rightarrow U^{\mathbb{C}} \\
L(\omega, \tilde{\omega})=K_{\omega} \tilde{\omega}+\frac{1}{4 \lambda^{2}} h\left(K_{\omega} \tilde{\omega}, e_{2}\right) e_{1}+\frac{1}{4 \lambda^{2}} h\left(K_{\omega} \tilde{\omega}, e_{1}\right) e_{2},
\end{array}\right.
$$

where $U^{\mathbb{C}}:=\operatorname{span}\left\{u_{1}, \ldots, u_{r}\right\}$ over $\mathbb{C}$.

Proposition 11. The operator $L$ defined in (21) satisfies:

(1) For any vectors $x \in \mathcal{W}_{1}$ and $y \in \mathcal{W}_{2}$ we have

$$
h(L(x, y), L(x, y))=\frac{3 \mu^{2}}{4} h(x, x) h(y, y) ;
$$

(2) Given $x_{0}$ in $\mathcal{W}_{1}$ such that $h\left(x_{0}, x_{0}\right)=1$, we have that $L\left(x_{0},-\right)$ preserves norms in the sense that

$$
h\left(L\left(x_{0}, y\right), L\left(x_{0}, y\right)\right)=\frac{3}{2} \mu^{2} h(y, y), \forall y \in \mathcal{W}_{2}
$$

(3) Given $x_{0}$ a non-null vector, we have that $L\left(x_{0},-\right): \mathcal{W}_{2} \mapsto \mathcal{U}^{\mathbb{C}}$ is a bijective operator;

(4) For any $x, x^{\prime} \in \mathcal{W}_{1}, y, z \in \mathcal{W}_{2}$ we have that

$$
h\left(L(x, y), L\left(x^{\prime}, z\right)\right)+h\left(L\left(x^{\prime}, y\right), L(x, z)\right)=\frac{3}{2} \mu^{2} h\left(x, x^{\prime}\right) h(y, z) .
$$

Proof. (1) Take $W_{1}=W_{3}=\omega_{1}$ and $W_{2}=W_{4}=\omega_{2}$ in relation (19), where $\omega_{1}:=v+i T v \in \mathcal{W}_{1}$ and $\omega_{2}:=w-i T w \in \mathcal{W}_{2}$. Using the properties of $T$ in (15) and the fact that $\omega_{1}$ and $\omega_{2}$ are orthogonal, we obtain $h\left(L\left(\omega_{1}, \omega_{2}\right), L\left(\omega_{1}, \omega_{2}\right)\right)=\frac{3 \mu^{2}}{4} h\left(\omega_{1}, \omega_{1}\right) h\left(\omega_{2}, \omega_{2}\right)$.

(2) This property follows directly from the previously proved one.

(3) We linearize in the second argument in property (22), that is $y \rightsquigarrow y+z$, for $y, z \in \mathcal{W}_{2}$ and we get for arbitrary $x \in \mathcal{W}_{1}$

$$
h(L(x, y), L(x, z))=\frac{3}{4} \mu^{2} h(x, x) h(y, z) .
$$

Fix $x=x_{0}$, for $x_{0}$ arbitrarily chosen in $\mathcal{W}_{1}$, and write equation (24) once for $y=y_{1}$ and once for $y=y_{2}$. Assuming $L\left(x_{0}, y_{1}\right)=L\left(x_{0}, y_{2}\right)$, as $h$ is nondegenerate and $x_{0}$ is a non-null vector, we get that $L\left(x_{0},-\right)$ is injective. This gives $\operatorname{dim} \operatorname{Im}\left(L\left(x_{0},-\right)\right)=\operatorname{dim} \mathcal{W}_{2}=r$, but, as $\operatorname{dim} U^{\mathbb{C}}=r$, we obtain that $L$ is also surjective. 
(4) The property in (23) follows immediately by liniarizing in (24) for $x \rightsquigarrow$ $x+x^{\prime}, \forall x, x^{\prime} \in \mathcal{W}_{1}$.

Theorem 12. Let $M_{k}^{n}$ be a $\lambda$-isotropic affine hypersurface. Assume that $\lambda \neq 0$. Then either $n=2,5,8,14$ or 26 .

Proof. We assume that $n>2$. We can write out equation (22) for the elements of the bases. For more convenience, choose $\left\{e_{i}\right\}_{i=\{1, \ldots, r\}},\left\{f_{j}\right\}_{j=\{1, \ldots, r\}},\left\{g_{k}\right\}_{k=\{1, \ldots, r\}}$ bases for $\mathcal{U}, \mathcal{W}_{1}, \mathcal{W}_{2}$, respectively, and let $u=\sum_{i=1}^{r} u_{i} e_{i}, v=\sum_{j=1}^{r} v_{j} f_{j}$. With this choice, relation (22) becomes

$$
\left(u_{1}^{2}+\ldots+u_{r}^{2}\right)\left(v_{1}^{2}+\ldots+v_{r}\right)=z_{1}^{2}+\ldots+z_{r}^{2},
$$

where $L\left(e_{i}, f_{j}\right)=l_{i j}^{k} g_{k}$ and $z_{k}=\sum_{i, j=1}^{n} u_{i} v_{j} l_{i j}^{k}$. Equation (25) yields an r-square quadratic equation. Thus, we may apply now the theorem of Hurwitz and obtain $r=1,2,4,8$, which implies that $n=5,8,14,26$.

\section{ISOTROPIC AFFINE HYPERSPHERES}

From now on, we will assume that $M$ is a $\lambda$-isotropic affine hypersphere with $\lambda \neq 0$.

Proposition 13. Let $n \geq 3$ and $M^{n}$ be an $n$-dimensional affine $\lambda$-isotropic hypersphere in $\mathbb{R}^{n+1}$. Then $M^{n}$ is constant isotropic.

Proof. Let $e_{1}^{\prime}:=f_{1}, e_{2}^{\prime}:=f_{2}, e_{3}^{\prime}:=u_{1}, \ldots, e_{r+2}^{\prime}:=u_{r}, e_{r+3}^{\prime}:=\omega_{1}^{1}, \ldots, e_{2 r+2}^{\prime}:=$ $\omega_{1}^{r}, e_{2 r+3}^{\prime}:=\omega_{2}^{r}$. Then $\left\{e_{1}^{\prime}, \ldots, e_{n}^{\prime}\right\}$ is an orthogonal basis with $h\left(e_{i}^{\prime}, e_{j}^{\prime}\right)=\varepsilon_{i} \delta_{i j}, \varepsilon_{i}=$ \pm 1 . We denote by Ric the Ricci tensor of $M^{n}$ with respect to the affine metric $h$. As $M^{n}$ is an affine sphere, we have that the shape operator is a multiple of the identity, say $S=\varepsilon I$. Using as well the Gauss equation in (2), the apolarity condition in proposition 5 and the isotropy condition (4) we have

$$
\begin{aligned}
\operatorname{Ric}\left(e_{j}^{\prime}, e_{k}^{\prime}\right) & =\sum_{i=1}^{n} \varepsilon_{i} h\left(\hat{R}\left(e_{i}^{\prime}, e_{j}^{\prime}\right) e_{k}^{\prime}, e_{i}^{\prime}\right) \\
& =h\left(\varepsilon\left(\varepsilon_{j} \delta_{j k} e_{i}^{\prime}-\varepsilon_{i} \delta_{i k} e_{j}^{\prime}\right)-\left[K_{e_{i}^{\prime}}, K_{e_{j}^{\prime}}\right] e_{k}^{\prime}, e_{i}^{\prime}\right) \\
& =n \varepsilon \varepsilon_{j} \delta_{j k}-\varepsilon \varepsilon_{j} \delta_{j k}-\sum_{i} \varepsilon_{i} h\left(\left[K_{e_{i}}, K_{e_{j}}\right] e_{k}, e_{i}\right) .
\end{aligned}
$$

For $k \neq j$ we obtain

$$
\begin{aligned}
\operatorname{Ric}\left(e_{j}^{\prime}, e_{k}^{\prime}\right) & =-\sum_{i=1}^{n} \varepsilon_{i} h\left(\left[K_{e_{i}^{\prime}}, K_{e_{j}^{\prime}}\right] e_{k}^{\prime}, e_{i}^{\prime}\right) \\
& =\sum_{i=1}^{n} \varepsilon_{i} h\left(K\left(e_{i}^{\prime}, e_{j}^{\prime}\right), K\left(e_{i}^{\prime}, e_{k}^{\prime}\right)\right) \\
& =-\frac{1}{2} \sum_{i=1}^{n} \varepsilon_{i} h\left(K\left(e_{i}^{\prime}, e_{i}^{\prime}\right), K\left(e_{j}^{\prime}, e_{k}^{\prime}\right)\right) \\
& =0
\end{aligned}
$$


and for $k=j$

$$
\begin{aligned}
\operatorname{Ric}\left(e_{j}^{\prime}, e_{j}^{\prime}\right) & =\sum_{i=1}^{n} \varepsilon h\left(K\left(e_{i}^{\prime}, e_{j}^{\prime}\right), K\left(e_{i}^{\prime}, e_{j}^{\prime}\right)\right) \\
& =\sum_{i=1}^{n} \frac{1}{2}\left[-h\left(K\left(e_{j}^{\prime}, e_{j}^{\prime}\right), K\left(e_{i}^{\prime}, e_{i}^{\prime}\right)\right)+2 \lambda(p) \delta_{i j}+\lambda(p) \varepsilon_{i} \varepsilon_{j}\right] \\
& =\left(\frac{n}{2}+1\right) \varepsilon_{j} \lambda(p) .
\end{aligned}
$$

Since $n \geq 3$, by using the fact that the Levi-Civita connection on $M^{n}$ is torsion free and using the second Bianchi identity, we get that $\lambda$ is constant.

Similarly to [9], Proposition 3.6, we can prove the following:

Proposition 14. Let $M^{n}$ be an $n$-dimensional affine submanifold in $\mathbb{R}^{n+1}$. If $M^{n}$ is constant isotropic with $\lambda \neq 0$, then $M^{n}$ has parallel difference tensor.

Proof Since $M^{n}$ is constant isotropic, we have $\lambda=h(K(v, v), K(v, v))$ and by taking the derivative, we obtain $h\left(\hat{\nabla}_{\chi} K(v, v), K(v, v)\right)=0, \forall p \in M^{n}, \forall v, \chi \in$ $T_{p} M^{n}, h(v, v)=1$.

In the isotropy relation (4) we take $X_{1}=\hat{\nabla}_{\chi} v, X_{2}=X_{3}=X_{4}=v$ and obtain $h\left(K\left(\hat{\nabla}_{\chi} v, v\right), K(v, v)\right)=\lambda h\left(\hat{\nabla}_{\chi} v, v\right) h(v, v)=0$, for $h(v, v)=1$. This implies

$$
h((\hat{\nabla} K)(\chi, v, v), h(v, v))=0 .
$$

for any $v, \chi \in T_{p} M^{n}$ such that $h(v, v)=1$ and in particular, we have

$$
h((\hat{\nabla} K)(v, v, v), h(v, v))=0,
$$

Further on, we take the derivative with respect to some vector $U \in T_{p} M^{n}$ in equation (4) for $X_{1}=X_{2}=X_{3}=v, X_{4}=w$ and for $h(v, w)=0$ and obtain

$$
h((\hat{\nabla} K)(v, v, v), K(v, w))-h((\hat{\nabla} K)(v, v, w), K(v, v))=0 .
$$

As $\hat{\nabla} K$ is totally symmetric, using also (29) we have

$$
h((\hat{\nabla} K)(v, v, v,), K(v, w))=0,
$$

for any $v, \chi \in T_{p} M^{n}$ such that $h(v, v)=1$. We can write $K(v, K(v, v))=a v+b w$, for $v \in T_{p} M^{n}, w$ an $(n-1)$-dimensional tangent vector,$h(v, w)=0$. Since

$$
\left\{\begin{array}{l}
h(K(v, K(v, v)), v)=h(K(v, v), K(v, v))=\lambda, \\
h(K(v, K(v, v)), w)=b h(w, w)=0,
\end{array}\right.
$$

we get $a=\lambda, b=0$ so that $K(v, K(v, v))=\lambda v$. If we take $w=K(v, v)$ in equation (31) we get

$$
\lambda h((\hat{\nabla} K)(v, v, v), v)=0 .
$$

As $\lambda \neq 0$, using (32) and the symmetry of $\hat{\nabla} K$, we also have $\hat{\nabla} K=0$.

Proposition 15. Let $n \geq 3$ and $M^{n}$ be an $n$-dimensional $\lambda$-isotropic affine hypersphere in $\mathbb{R}^{n+1}$, such that $S=\varepsilon I$, with $\varepsilon$ constant. Assume that $\lambda \neq 0$. If $\mathbb{R}^{n+1}$ is endowed with an indefinite metric and $M^{n}$ is not totally geodesic, then $M^{n}$ is a locally symmetric space and $\lambda=-\frac{1}{2} \varepsilon$.

Proof. From the previous propositions we conclude that $\hat{\nabla} K=0$. Hence, by the Gauss equation we have $\hat{\nabla} R=0$, which means that $M^{n}$ is a locally symmetric space. Using the Ricci identity, from $\hat{\nabla} K=0$ we also have $\hat{R} \cdot h=0$, that is

$$
\hat{R}(X, Y) K(Z, W)-K(\hat{R}(X, Y) Z, W)-K(Z, \hat{R}(X, Y) W)=0,
$$


for $X, Y, Z, W$ tangent vector fields. If we take $X=Z=W=f_{1}, Y=f_{2}$, it implies

$$
\hat{R}\left(f_{1}, f_{2}\right) K\left(f_{1}, f_{1}\right)=2 K\left(\hat{R}\left(f_{1}, f_{2}\right) f_{1}, f_{1}\right)
$$

and then, from Gauss equation we have

$$
R\left(f_{1}, f_{2}\right) f_{1}=-(\varepsilon+2 \lambda) f_{2},
$$

which together with (34) implies $\varepsilon+2 \lambda=0$.

Proposition 16. Let $n \geq 3, f_{1}: M_{1}^{n} \rightarrow \mathbb{R}^{n}$ and $f_{2}: M_{1}^{n} \rightarrow \mathbb{R}^{n}$ be $n$-dimensional $\lambda$-isotropic affine hypersphere in $\mathbb{R}^{n+1}$, such that $S_{1}=S_{2}=\varepsilon I$, with $\varepsilon= \pm 1$ constant. Let $p_{1} \in M_{1}$ and $p_{2} \in M_{2}$ and assume that there exists an isometry $A: T_{p_{1}} M_{1} \rightarrow T_{p_{2}} M_{2}$ such that

$$
A K_{1}(v, w)=K_{2}(A v, A w)
$$

i.e. A preserves the difference tensor. Then there exists a local isometry $F$ : $\left(M_{1}, h_{1}\right) \rightarrow\left(M_{2}, h_{2}\right)$ such that

$$
d F\left(K_{1}(X, Y)\right)=K_{2}(d F(X), d F(Y)),
$$

for any vector fields $X, Y$ on $M_{1}$. Moreover the immersions $f_{1}$ and $f_{2} \circ F$ are locally congruent.

Proof. From the previous propositions we know that $\lambda$ is a constant, and that with respect to the Levi Civita connection $M_{1}$ and $M_{2}$ are locally symmetric spaces whose difference tensor is parallel with respect to the Levi Civita connection.

We take $p_{1} \in M_{1}$ and we take a basis $\left\{e_{1}^{\prime}, \ldots, e_{n}^{\prime}\right\}$ of $T_{p_{1}} M_{1}$. As $A$ is an isometry we take as basis of $T_{p_{2}} M_{2}$ the vectors $\left\{A e_{1}^{\prime}, \ldots, A e_{n}^{\prime}\right\}$. By the initial conditions we have that

$$
\begin{aligned}
& h_{1}\left(e_{i}^{\prime}, e_{j}^{\prime}\right)=h_{2}\left(A e_{i}^{\prime} A e_{j}^{\prime}\right) \quad \text { (isometry) } \\
& h_{1}\left(K_{1}\left(e_{i}^{\prime}, e_{j}^{\prime}\right), e_{k}^{\prime}\right)=h_{2}\left(A K_{1}\left(e_{i}^{\prime}, e_{j}^{\prime}\right), A e_{k}^{\prime}\right)=h_{2}\left(K_{2}\left(A e_{i}^{\prime}, A e_{j}^{\prime}\right), A e_{k}^{\prime}\right) .
\end{aligned}
$$

We now extend $\left\{e_{1}^{\prime}, \ldots, e_{n}^{\prime}\right\}$ to a local differential basis $\left\{X_{1}, \ldots, X_{n}\right\}$ by parallel translation along geodesics with respect to the Levi Civita connection of the affine metric. In the same way we extend $\left\{A e_{1}^{\prime}, \ldots, A e_{n}^{\prime}\right\}$ to local vector fields $\left\{Y_{1}, \ldots, Y_{n}\right\}$. As the difference tensors are parallel, we have that the components of the difference tensor stay constant along geodesics. Therefore by construction, we have that

$$
\begin{aligned}
& h_{1}\left(X_{i}, X_{j}\right)=h_{2}\left(Y_{i}, Y_{j}\right), \\
& h_{1}\left(K_{1}\left(X_{i}, X_{j}\right), X_{k}\right)=h_{2}\left(K_{2}\left(Y_{i}, Y_{j}\right), Y_{k}\right)
\end{aligned}
$$

Hence by the lemma of Cartan, see [7] we know that there exists a local isometry $F$ such that $d F\left(X_{i}\right)=Y_{i}$. In order to complete the proof it is now sufficent to apply Theorem 9 .

So in order to complete the classification it is now sufficient to determine, up to isometries, the possible forms of the difference tensor and for each of those forms obtained to determine an explicit example of an affine hypersphere with isotropic difference tensors. This is done explicitly for the 4 remaining dimensions $5,8,14$ and 26 in the next sections. 


\section{AFFINE HYPERSPHERES OF DIMENSION 5}

5.1. The form of $L, \operatorname{dim} \mathcal{U}=1$. We start with $\omega=v+i T v \in \mathcal{W}_{1}$, a vector of length 2. As the length of $\omega$ is 2 it follows that $v$ has unit length and is orthogonal to $T v$. So we can take $\omega_{1}^{1}=v$ and $\omega_{2}^{1}=T v$. Note that by the properties of $L$ we have that $L(v+i T v, v-i T v)$ is a real vector in $\mathcal{U}^{\mathbb{C}}$ whose square length is $3 \mu^{2}$. Hence we can pick a unit vector $u_{1}$ in $U$ such that

$$
L(v+i T v, v-i T v)=\sqrt{3} \mu u_{1} .
$$

By the properties of $L$ this implies that

$$
L\left(\omega_{1}^{1}, \omega_{1}^{1}\right)=\frac{\sqrt{3}}{2} \mu u_{1}
$$

From the properties of $T$ we see $L\left(\omega_{1}^{1}, \omega_{2}^{1}\right)=0$ and $L\left(\omega_{1}^{1}, \omega_{1}^{1}\right)=L\left(\omega_{2}^{1}, \omega_{2}^{1}\right)$, hence $L$ is completely determined. Therefore $L$ and also $K$ are completely determined and the signature of the metric, if necessary after replacing $\xi$ by $-\xi$ in order to make $\lambda>0$, equals 2 .

\subsection{A canonical example.}

We consider $\mathbb{R}^{6}=s(3)$ as the set of all symmetric $3 \times 3$ matrices and we take as hypersurface $M$ those symmetric matrices with determinant 1 . We define an action $\sigma$ of $S L(3, \mathbb{R})$ on $M$ as follows

$$
\sigma: S L(3, \mathbb{R}) \times \rightarrow M, \text { such that }(g, p) \mapsto \sigma_{g}(p)=g p g^{T}
$$

Note that $M$ has two connected components and that the action is transitive on each of the connected components. The connected component of $I$ has been studied in [2] where it was shown that it gives a positive definite isotropic affine hypersurface. It also appears in [8]. Here we are interested in the component of the matrix $A=\left(\begin{array}{ccc}1 & 0 & 0 \\ 0 & -1 & 0 \\ 0 & 0 & -1\end{array}\right)$, which we denote by $M_{1}$. So $M_{1}=\left\{g A g^{T} \mid g \in S L(3, \mathbb{R})\right\}$. The isotropy group of $A$ consists of the matrices $g$ of determinant 1 such that $g A g^{T}=A$. This Lie group is congruent to $S O(2,1)$ and therefore, by Theorem 9.2 of [3], we know that $M_{1}$ is locally isometric with $\frac{S L(3, \mathbb{R})}{S O(2,1)}$.

Note that, of course, every element of $S L(3, \mathbb{R})$ acts at the same time also on $s(3)$ and that this action belongs to $S L(6, \mathbb{R})$, see [13]. This implies that $M_{1}$ is at the same time an homogeneous affine hypersurface and by Proposition 6 an equiaffine sphere centered at the origin.

In order to determine the tangent space at $p=g A g^{T}$, we look at the curves in $M_{1}$

$$
\gamma(s)=g e^{s X} A e^{s X^{T}} g^{T}
$$

These are indeed curves in $M_{1}$, provided that $e^{s X} \in S L(3, \mathbb{R})$ or, equivalently, provided that $\operatorname{Tr} X=0$. Note that $\gamma^{\prime}(s)=g e^{s X}\left(X A+A X^{T}\right) e^{s X^{T}} g^{T}$, where $v=\left(X A+A X^{T}\right)$ is a symmetric matrix. So by using a dimension argument we see that the tangent space is given by

$$
\left\{g v g^{T} \mid v=2 X A, X A=A X^{T}, \operatorname{Tr} X=0, X \in \mathbb{R}^{3 \times 3}\right\}=T_{p} M_{1} .
$$


Working now at the point $A$, taking $g=I$ and $X \in s o(2,1)=\left\{X \in \mathbb{R}^{3 \times 3} \mid \operatorname{Tr} X=\right.$ $\left.0, X A=A X^{T}\right\}$ we see that

$$
\begin{aligned}
\nabla_{\gamma^{\prime}(s)} \gamma^{\prime}(s)+h\left(\gamma^{\prime}, \gamma^{\prime}\right) \gamma & =\gamma^{\prime \prime}(s) \\
& =e^{s X}\left(4 X^{2} A\right) e^{s X^{T}} \\
& =e^{s X}\left(\left(4 X^{2}-\frac{4}{3} \operatorname{Tr}\left(X^{2}\right) I\right) A\right) e^{s X^{T}}+\frac{4}{3} \operatorname{Tr}\left(X^{2}\right) e^{s X} A e^{s X^{T}} \\
& =e^{s X}\left(\left(4 X^{2}-\frac{4}{3} \operatorname{Tr}\left(X^{2}\right) I\right) A\right) e^{s X^{T}}+\frac{4}{3} \operatorname{Tr}\left(X^{2}\right) \gamma(s)
\end{aligned}
$$

As the matrix $\left(4 X^{2}-\frac{4}{3} \operatorname{Tr}\left(X^{2}\right) I\right)$ commutes with $A$, we can decompose the above expression into a tangent part and a part in the direction of the affine normal given by the position vector, and therefore we find that

$$
h\left(\gamma^{\prime}(s), \gamma^{\prime}(s)\right)=\frac{4}{3} \operatorname{Tr}\left(X^{2}\right) .
$$

So we see that $s$ is a constant length parametrisation of the curve $\gamma$ and therefore we have that $h\left(\gamma^{\prime}, \hat{\nabla}_{\gamma^{\prime}} \gamma^{\prime}\right)=0$ and

$$
h\left(\gamma^{\prime}, \nabla_{\gamma^{\prime}} \gamma^{\prime}\right)=h\left(\gamma^{\prime}, K\left(\gamma^{\prime}, \gamma^{\prime}\right)\right)
$$

In addition, we have

$$
\begin{aligned}
\gamma^{\prime \prime \prime}(s) & =\nabla_{\gamma^{\prime}(s)} \nabla_{\gamma^{\prime}(s)} \gamma^{\prime}(s)+h\left(\gamma^{\prime}, \gamma^{\prime}\right) \gamma^{\prime}+h\left(\gamma^{\prime}, K\left(\gamma^{\prime}, \gamma^{\prime}\right) \gamma\right. \\
& =e^{s X}\left(8 X^{3} A\right) e^{s X^{T}} \\
& =e^{s X}\left(\left(8 X^{3}-\frac{8}{3} \operatorname{Tr}\left(X^{3}\right) I\right) A\right) e^{s X^{T}}+\frac{8}{3} \operatorname{Tr}\left(X^{3}\right) \gamma(s) .
\end{aligned}
$$

So therefore working at $s=0$ and writing $v=2 X A$ as tangent vector, we obtain that

$$
\begin{aligned}
& h(v, v)=\frac{4}{3} \operatorname{Tr}\left(X^{2}\right), \\
& h(v, K(v, v))=\frac{8}{3} \operatorname{Tr} X^{3} .
\end{aligned}
$$

Linearising the above expressions, i.e. writing $v=\alpha_{1} v_{1}+\alpha_{2} v_{2}$, respectively $v=$ $\alpha_{1} v_{1}+\alpha_{2} v_{2}+\alpha_{3} v_{3}$, for $v=2 X_{i} A, i=1,2,3$, and looking at the coefficient of $\alpha_{1} \alpha_{2}$, respectively $\alpha_{1} \alpha_{2} \alpha_{3}$, we obtain that

$$
\begin{aligned}
h\left(v_{1}, v_{2}\right) & =\frac{4}{3} \operatorname{Tr}\left(X_{1} X_{2}\right)=\frac{4}{3} \operatorname{Tr}\left(X_{2} X_{1}\right) \\
6 h\left(K\left(v_{1}, v_{2}\right), v_{3}\right) & =\frac{8}{3}\left(\operatorname{Tr} X_{1} X_{2} X_{3}+\operatorname{Tr} X_{3} X_{1} X_{2}+\operatorname{Tr} X_{2} X_{3} X_{1}+\operatorname{Tr} X_{1} X_{3} X_{2}+\operatorname{Tr} X_{3} X_{2} X_{1}+\operatorname{Tr} X_{2} X_{1} X_{3}\right) \\
& =8\left(\operatorname{Tr} X_{1} X_{2} X_{3}+\operatorname{Tr} X_{2} X_{1} X_{3}\right) .
\end{aligned}
$$

So we see that

$$
K\left(v_{1}, v_{2}\right)=2\left(X_{1} X_{2}+X_{2} X_{1}-\frac{2}{3} \operatorname{Tr}\left(X_{1} X_{2}\right) I\right) A .
$$

Indeed, we have that $\left(X_{1} X_{2}+X_{2} X_{1}-\frac{2}{3} \operatorname{Tr}\left(X_{1} X_{2}\right) I\right)$ has vanishing trace, commutes with $A$ and therefore $K\left(v_{1}, v_{2}\right)$ is indeed the unique tangent vector such that

$$
h\left(K\left(v_{1}, v_{2}\right), v_{3}\right)=\frac{4}{3}\left(\operatorname{Tr}\left(X_{1} X_{2} X_{3}\right)+\operatorname{Tr}\left(X_{2} X_{1} X_{3}\right)\right) .
$$

As by Cayley Hamilton, for a matrix $X$ with vanishing trace, we have that $X^{3}=$ $1 / 2 \operatorname{Tr}\left(X^{2}\right) X+\operatorname{det}(X) I$, we deduce that

$$
\operatorname{Tr} X^{4}=\frac{1}{2}\left(\operatorname{Tr} X^{2}\right)^{2},
$$

and therefore we have that

$$
\begin{aligned}
h(K(v, v), K(v, v)) & =\frac{4}{3} \operatorname{Tr}\left(2 X^{2}-\frac{2}{3} \operatorname{Tr} X^{2} I\right)^{2} \\
& =\frac{4}{3}\left(4 \operatorname{Tr} X^{4}+\frac{4}{9}\left(\operatorname{Tr} X^{2}\right)^{2} \operatorname{Tr} I-\frac{8}{3}\left(\operatorname{Tr} X^{2}\right)^{2}\right) \\
& =\frac{8}{9}\left(\operatorname{Tr} X^{2}\right)^{2} \\
& =\frac{1}{2}(h(v, v))^{2} .
\end{aligned}
$$


Hence $M_{1}$ is isotropic with positive $\lambda$. A straightforward computation also shows that the index of the metric is 2 . Combining therefore the results in this section with Proposition 16 and the classification result of O. Birembaux and M. Djoric, see [2] in the positive definite case, we get the following theorem:

Theorem 17. Let $M$ be a 5-dimensional $\lambda$-isotropic affine hypersphere, with $\lambda \neq 0$. Therefore, if necessary, by changing the sign of the affine normal, we may assume that $\lambda$ is positive. Then either

(1) the metric is positive definite, $M$ is isometric with $\frac{S L(3, \mathbb{R})}{S O(3)}$ and $M$ is affine congruent to an open part of the hypersurface $\left\{g g^{T} \mid g \in S L(3, \mathbb{R})\right\}$ of $\mathbb{R}^{6} \equiv$ $s(3) \subset \mathbb{R}^{3 \times 3}$, or

(2) the metric has signature 2, $M$ is isometric with $\frac{S L(3, \mathbb{R})}{S O(2,1)}$ and $M$ is affine congruent to an open part of the hypersurface $\left\{g A g^{T} \mid g \in S L(3, \mathbb{R})\right\}$, where $A=\left(\begin{array}{ccc}1 & 0 & 0 \\ 0 & -1 & 0 \\ 0 & 0 & -1\end{array}\right)$, of $\mathbb{R}^{6} \equiv s(3) \subset \mathbb{R}^{3 \times 3}$.

\section{AfFine HYPERSPHERES OF DIMENSION 8}

6.1. The form of $L, \operatorname{dim} \mathcal{U}=2$.

Let $W=\operatorname{span}\left\{\omega_{1}^{1}, \omega_{2}^{1}, \omega_{1}^{2}, \omega_{2}^{2}\right\}$ and $\mathcal{W}_{1}=\operatorname{span}\left\{\omega_{1}^{1}+i \omega_{2}^{1}, \omega_{1}^{2}+i \omega_{2}^{2}\right\}, \mathcal{W}_{2}=\operatorname{span}\left\{\omega_{1}^{1}-\right.$ $\left.i \omega_{2}^{1}, \omega_{1}^{2}-i \omega_{2}^{2}\right\}$. Remark that all the bases are orthogonal and in addition

$$
\begin{aligned}
& h\left(\omega_{1}^{1}+i \omega_{2}^{1}, \omega_{1}^{1}+i \omega_{2}^{1}\right)=h\left(\omega_{1}^{2}+i \omega_{2}^{2}, \omega_{1}^{2}+i \omega_{2}^{2}\right)=2, \\
& h\left(\omega_{1}^{1}-i \omega_{2}^{1}, \omega_{1}^{1}-i \omega_{2}^{1}\right)=h\left(\omega_{1}^{2}-i \omega_{2}^{2}, \omega_{1}^{2}-i \omega_{2}^{2}\right)=-2 .
\end{aligned}
$$

Then, straightforward computations lead to

$$
\left\{\begin{array}{l}
L\left(\omega_{1}^{1}+i \omega_{2}^{1}, \omega_{1}^{1}-i \omega_{2}^{1}\right)=2 L\left(\omega_{1}^{1}, \omega_{1}^{1}\right) \\
L\left(\omega_{1}^{1}+i \omega_{2}^{1}, \omega_{1}^{2}-i \omega_{2}^{2}\right)=2 L\left(\omega_{1}^{1}, \omega_{1}^{2}\right)-2 i L\left(\omega_{1}^{1}, \omega_{2}^{2}\right) \\
L\left(\omega_{1}^{2}+i \omega_{2}^{2}, \omega_{1}^{1}-i \omega_{2}^{1}\right)=2 L\left(\omega_{1}^{1}, \omega_{1}^{2}\right)+2 i L\left(\omega_{1}^{1}, \omega_{2}^{2}\right) \\
L\left(\omega_{1}^{2}+i \omega_{2}^{2}, \omega_{1}^{2}-i \omega_{2}^{2}\right)=2 L\left(\omega_{1}^{2}, \omega_{1}^{2}\right)
\end{array}\right.
$$

Notice that the vector $L\left(\omega_{1}^{1}+i \omega_{2}^{1}, \omega_{1}^{1}-i \omega_{2}^{1}\right)$ is a real vector of length $3 \mu^{2}$. So we can pick $u_{1} \in \mathcal{U}, h\left(u_{1}, u_{1}\right)=1$ such that

$$
L\left(\omega_{1}^{1}+i \omega_{2}^{1}, \omega_{1}^{1}-i \omega_{2}^{1}\right)=\sqrt{3} \mu u_{1} .
$$

With this choice, from property (24) we obtain that $L\left(\omega_{1}^{1}+i \omega_{2}^{1}, \omega_{1}^{2}-i \omega_{2}^{2}\right)$ is orthogonal to $u_{1}$. Moreover as its length is a real number, we must have that $\operatorname{Re}\left(L\left(\omega_{1}^{1}+i \omega_{2}^{1}, \omega_{1}^{2}-i \omega_{2}^{2}\right)\right.$ and $\operatorname{Im}\left(L\left(\omega_{1}^{1}+i \omega_{2}^{1}, \omega_{1}^{2}-i \omega_{2}^{2}\right)\right.$ are orthogonal to each other. As they are also both orthogonal to $u_{1}$, one of them has to vanish. Therefore, we get two cases:

Case II-1. $\operatorname{Re}\left(L\left(\omega_{1}^{1}+i \omega_{2}^{1}, \omega_{1}^{2}-i \omega_{2}^{2}\right)\right)=0$

Now we obtain that $L\left(\omega_{1}^{1}, \omega_{1}^{2}\right)=0$ and $L\left(\omega_{1}^{1}+i \omega_{2}^{1}, \omega_{1}^{2}-i \omega_{2}^{2}\right)$ is an imaginary vector of length $3 \mu^{2}$, orthogonal to $u_{1}$. Thus, we can pick $u_{2} \in \mathcal{U}$ in the direction of $L\left(\omega_{1}^{1}+i \omega_{2}^{1}, \omega_{1}^{2}-i \omega_{2}^{2}\right)$ such that $h\left(u_{2}, u_{2}\right)=-1$ and such that

$$
L\left(\omega_{1}^{1}+i \omega_{2}^{1}, \omega_{1}^{2}-i \omega_{2}^{2}\right)=i \sqrt{3} \mu u_{2} .
$$

Consider now $L\left(\omega_{1}^{2}+i \omega_{2}^{2}, \omega_{1}^{2}-i \omega_{2}^{2}\right)$. It is a real vector orthogonal to $u_{2}$, of length $3 \mu^{2}$ and thus we can write $L\left(\omega_{1}^{2}+i \omega_{2}^{2}, \omega_{1}^{2}-i \omega_{2}^{2}\right)= \pm \sqrt{3} \mu u_{1}$.

Furthermore, from (35), (37) and Proposition 11- (4), we obtain

$$
\begin{aligned}
3 \mu^{2} & =-h\left(L\left(\omega_{1}^{1}+i \omega_{2}^{1}, \omega_{1}^{2}-i \omega_{2}^{2}\right), L\left(\omega_{1}^{2}+i \omega_{2}^{2}, \omega_{1}^{1}-i \omega_{2}^{1}\right)\right) \\
& =h\left(L\left(\omega_{1}^{1}+i \omega_{2}^{1}, \omega_{1}^{1}-i \omega_{2}^{1}\right), L\left(\omega_{1}^{2}+i \omega_{2}^{2}, \omega_{1}^{2}-i \omega_{2}^{2}\right)\right) \\
& =\sqrt{3} \mu h\left(u_{1}, L\left(\omega_{1}^{2}+i \omega_{2}^{2}, \omega_{1}^{2}-i \omega_{2}^{2}\right)\right) .
\end{aligned}
$$


So we get that $L\left(\omega_{1}^{2}+i \omega_{2}^{2}, \omega_{1}^{2}-i \omega_{2}^{2}\right)=\sqrt{3} \mu u_{1}$. In this case, the signature of the metric is 4 .

Case II-2. $\operatorname{Im}\left(L\left(\omega_{1}^{1}+i \omega_{2}^{1}, \omega_{1}^{2}-i \omega_{2}^{2}\right)\right)=0$

Reasoning in a similar way, we choose $u_{2} \in \mathcal{U}$ a real vector in the direction of $L\left(\omega_{1}^{1}+i \omega_{2}^{1}, \omega_{1}^{2}-i \omega_{2}^{2}\right)$, with $h\left(u_{2}, u_{2}\right)=1$ such that $L\left(\omega_{1}^{1}+i \omega_{2}^{1}, \omega_{1}^{2}-i \omega_{2}^{2}\right)=\sqrt{3} \mu u_{2}$. We find $L\left(\omega_{1}^{2}+i \omega_{2}^{2}, \omega_{1}^{2}-i \omega_{2}^{2}\right)=-\sqrt{3} \mu u_{1}$ and in this case the signature of the metric is 3 .

\subsection{Two canonical examples.}

First we consider $\mathbb{R}^{9}$ as the set of Hermitian symmetric matrics $Y \in \mathbb{C}^{3 \times 3}$. We take as hypersurface $M$ those Hermitian symmetric matrices with determinant 1 . We define an action $\sigma$ of $S L(3, \mathbb{C})$ on $M$ as follows

$$
\sigma: S L(3, \mathbb{C}) \times M \rightarrow M, \text { such that }(g, p) \mapsto \sigma_{g}(p)=g p \bar{g}^{T} .
$$

Note that $M$ has two connected components and that the action is transitive on each of the connected components. The connected component of $I$ has been studied in [2], where it was shown that it gives a positive definite isotropic affine hypersurface. It also appears in [8]. Here we are interested in the component of the matrix $A=\left(\begin{array}{ccc}1 & 0 & 0 \\ 0 & -1 & 0 \\ 0 & 0 & -1\end{array}\right)$, which we denote by $M_{1}$. So $M_{1}=\left\{g A \bar{g}^{T} \mid g \in S L(3, \mathbb{R})\right\}$.

The isotropy group consists of the matrices $g$ of determinant 1 such that $g A \bar{g}^{T}=A$. This Lie group is congruent to $S U(2,1)$ and therefore, by Theorem 9.2 of [3], we know that $M_{1}$ is locally isometric with $\frac{S L(3, \mathbb{C})}{S U(2,1)}$.

Note that of course every element of $S L(3, \mathbb{C})$ acts at the same time also on $\mathbb{R}^{9}$ in a linear way and that, therefore, this action belongs to $G L(9, \mathbb{R})$. A straightforward computation shows that this action actually belongs to $S L(9, \mathbb{R})$. This implies that $M_{1}$ is at the same time an homogeneous affine hypersurface and, by Proposition 6 , an equiaffine sphere centered at the origin. So, in order to determine the properties of $M_{1}$, it is sufficient to look at a single point.

In order to determine the tangent space at the point $p=g A \bar{g}^{T}$, we look at the curves in $M_{1}$

$$
\gamma(s)=g e^{s X} A e^{s \bar{X}^{T}} \bar{g}^{T} .
$$

These are indeed curves in $M_{1}$ provided that $e^{s X} \in S L(3, \mathbb{C})$ or equivalently provided that $\operatorname{Tr} X=0$. Note that $\gamma^{\prime}(s)=g e^{s X}\left(X A+A \bar{X}^{T}\right) e^{s \bar{X}^{T}} \bar{g}^{T}$, where $v=\left(X A+A \bar{X}^{T}\right)$ is a Hermitian symmetric matrix. So by using a dimension argument we see that the tangent space is given by

$$
\left\{g v \bar{g}^{T} \mid v=2 X A, X A=A \bar{X}^{T}, \operatorname{Tr} X=0, X \in \mathbb{C}^{3 \times 3}\right\}=T_{p} M_{1} .
$$

Working now at the point $A$, taking $g=I$ and $X \in s u(2,1)=\left\{X \in \mathbb{C}^{3 \times 3} \mid \operatorname{Tr} X=\right.$ $\left.0, X A=A \bar{X}^{T}\right\}$ we see that

$$
\begin{aligned}
\nabla_{\gamma^{\prime}(s)} \gamma^{\prime}(s)+h\left(\gamma^{\prime}, \gamma^{\prime}\right) \gamma & =\gamma^{\prime \prime}(s) \\
& =e^{s X}\left(4 X^{2} A\right) e^{s X^{T}} \\
& =e^{s X}\left(\left(4 X^{2}-\frac{4}{3} \operatorname{Tr}\left(X^{2}\right) I\right) A\right) e^{s \bar{X}^{T}}+\frac{4}{3} \operatorname{Tr}\left(X^{2}\right) e^{s X} A e^{s \bar{X}^{T}} \\
& =e^{s X}\left(\left(4 X^{2}-\frac{4}{3} \operatorname{Tr}\left(X^{2}\right) I\right) A\right) e^{s \bar{X}^{T}}+\frac{4}{3} \operatorname{Tr}\left(X^{2}\right) \gamma(s) .
\end{aligned}
$$

As the matrix $\left(4 X^{2}-\frac{4}{3} \operatorname{Tr}\left(X^{2}\right) I\right)$ commutes with $A$, we can decompose the above expression into a tangent part and a part in the direction of the affine normal given by the position vector, and therefore we find that

$$
h\left(\gamma^{\prime}(s), \gamma^{\prime}(s)\right)=\frac{4}{3} \operatorname{Tr}\left(X^{2}\right) .
$$


So we see that $s$ is a constant length parametrisation of the curve $\gamma$ and therefore we have that $h\left(\gamma^{\prime}, \hat{\nabla}_{\gamma^{\prime}} \gamma^{\prime}\right)=0$ and

$$
h\left(\gamma^{\prime}, \nabla_{\gamma^{\prime}} \gamma^{\prime}\right)=h\left(\gamma^{\prime}, K\left(\gamma^{\prime}, \gamma^{\prime}\right)\right)
$$

As

$$
\begin{aligned}
\gamma^{\prime \prime \prime}(s) & =\nabla_{\gamma^{\prime}(s)} \nabla_{\gamma^{\prime}(s)} \gamma^{\prime}(s)+h\left(\gamma^{\prime}, \gamma^{\prime}\right) \gamma^{\prime}+h\left(\gamma^{\prime}, K\left(\gamma^{\prime}, \gamma^{\prime}\right) \gamma\right. \\
& =e^{s X}\left(8 X^{3} A\right) e^{s \bar{X}^{T}} \\
& =e^{s X}\left(\left(8 X^{3}-\frac{8}{3} \operatorname{Tr}\left(X^{3}\right) I\right) A\right) e^{s \bar{X}^{T}}+\frac{8}{3} \operatorname{Tr}\left(X^{3}\right) \gamma(s),
\end{aligned}
$$

working at $s=0$ and writing $v=2 X A$ as tangent vector, we have that

$$
\begin{aligned}
& h(v, v)=\frac{4}{3} \operatorname{Tr}\left(X^{2}\right), \\
& h(v, K(v, v))=\frac{8}{3} \operatorname{Tr} X^{3} .
\end{aligned}
$$

Linearising the above expressions, i.e. writing $v=\alpha_{1} v_{1}+\alpha_{2} v_{2}$, respectively $v=$ $\alpha_{1} v_{1}+\alpha_{2} v_{2}+\alpha_{3} v_{3}$, for $v=2 X_{i} A, i=1,2,3$, and looking at the coefficient of $\alpha_{1} \alpha_{2}$, respectively $\alpha_{1} \alpha_{2} \alpha_{3}$, we obtain that

$$
\begin{aligned}
h\left(v_{1}, v_{2}\right) & =\frac{4}{3} \operatorname{Tr}\left(X_{1} X_{2}\right)=\frac{4}{3} \operatorname{Tr}\left(X_{2} X_{1}\right), \\
6 h\left(K\left(v_{1}, v_{2}\right), v_{3}\right) & =\frac{8}{3}\left(\operatorname{Tr} X_{1} X_{2} X_{3}+\operatorname{Tr} X_{3} X_{1} X_{2}+\operatorname{Tr} X_{2} X_{3} X_{1}+\operatorname{Tr} X_{1} X_{3} X_{2}+\operatorname{Tr} X_{3} X_{2} X_{1}+\operatorname{Tr} X_{2} X_{1} X_{3}\right) \\
& =8\left(\operatorname{Tr} X_{1} X_{2} X_{3}+\operatorname{Tr} X_{2} X_{1} X_{3}\right) .
\end{aligned}
$$

So we see that

$$
K\left(v_{1}, v_{2}\right)=2\left(X_{1} X_{2}+X_{2} X_{1}-\frac{2}{3} \operatorname{Tr}\left(X_{1} X_{2}\right) I\right) A .
$$

Indeed, we have that $\left(X_{1} X_{2}+X_{2} X_{1}-\frac{2}{3} \operatorname{Tr}(X Y) I\right)$ has vanishing trace, commutes with $A$ and therefore $K\left(v_{1}, v_{2}\right)$ is indeed the unique tangent vector such that

$$
h\left(K\left(v_{1}, v_{2}\right), v_{3}\right)=\frac{4}{3}\left(\operatorname{Tr}\left(X_{1} X_{2} X_{3}\right)+\operatorname{Tr}\left(X_{2} X_{1} X_{3}\right)\right) .
$$

As by Cayley Hamilton for a matrix $X$ with vanishing trace we have that $X^{3}=$ $1 / 2 \operatorname{Tr}\left(X^{2}\right) X+\operatorname{det}(X) I$, we deduce that

$$
\operatorname{Tr} X^{4}=\frac{1}{2} \operatorname{Tr} X^{2},
$$

and therefore, we have that

$$
\begin{aligned}
h(K(v, v), K(v, v)) & =\frac{4}{3} \operatorname{Tr}\left(2 X^{2}-\frac{2}{3} \operatorname{Tr} X^{2} I\right)^{2} \\
& =\frac{4}{3}\left(4 \operatorname{Tr} X^{4}+\frac{4}{9}\left(\operatorname{Tr} X^{2}\right)^{2} \operatorname{Tr} I-\frac{8}{3}\left(\operatorname{Tr} X^{2}\right)^{2}\right) \\
& =\frac{8}{9}\left(\operatorname{Tr} X^{2}\right)^{2} \\
& =\frac{1}{2}(h(v, v))^{2} .
\end{aligned}
$$

Hence $M_{1}$ is isotropic with positive $\lambda$. A straightforward computation also shows that the index of the metric is 4 .

Next, we consider $\mathbb{R}^{9}=\mathbb{R}^{3 \times 3}$. We take as hypersurface $M_{2}$ those matrices with determinant 1 . We define an action $\sigma$ of $S L(3, \mathbb{R})$ on $M_{2}$ as follows

$$
\sigma: S L(3, \mathbb{R}) \times M_{2} \rightarrow M_{2} \text {, such that }(g, p) \mapsto \sigma_{g}(p)=g p .
$$

The isotropy group of the identity matrix consists only of the identity matrix. Therefore, by Theorem 9.2 of [3] we know that $M_{2}$ is locally isometric with $S L(3, \mathbb{R})$.

Note that, of course, every element of $S L(3, \mathbb{R})$ acts at the same time also on $\mathbb{R}^{9}$ in a linear way and that therefore this action belongs to $G L(9, \mathbb{R})$. A straightforward computation shows that this action actually belongs to $S L(9, \mathbb{R})$. This implies that $M_{2}$ is at the same time an homogeneous affine hypersurface and, by Proposition 6 , an equiaffine sphere centered at the origin. So in order to determine the properties of $M_{2}$ it is sufficient to look at a single point. 
In order to determine the tangent space at a point $p$, we look at the curves in $M_{2}$

$$
\gamma(s)=e^{s X} p
$$

These are indeed curves in $M_{2}$, provided that $e^{s X} \in S L(3, \mathbb{R})$ or equivalently, provided that $\operatorname{Tr} X=0$. Note that $\gamma^{\prime}(s)=e^{s X} X p$, so by using a dimension argument we see that the tangent space is given by

$$
\left\{X p \mid \operatorname{Tr} X=0, X \in \mathbb{R}^{3 \times 3}\right\}=T_{p} M_{2} .
$$

Working now at the point $I$ and $X \in \operatorname{sl}(3, \mathbb{R})=\left\{X \in \mathbb{R}^{3 \times 3} \mid \operatorname{Tr} X=0\right\}$, we see that

$$
\begin{aligned}
\nabla_{\gamma^{\prime}(s)} \gamma^{\prime}(s)+h\left(\gamma^{\prime}, \gamma^{\prime}\right) \gamma & =\gamma^{\prime \prime}(s) \\
& =e^{s X} X^{2} \\
& =e^{s X}\left(X^{2}-\frac{1}{3} \operatorname{Tr}\left(X^{2}\right) I\right)+\frac{1}{3} \operatorname{Tr}\left(X^{2}\right) e^{s X} \\
& =e^{s X}\left(X^{2}-\frac{1}{3} \operatorname{Tr}\left(X^{2}\right) I\right)+\frac{1}{3} \operatorname{Tr}\left(X^{2}\right) \gamma(s)
\end{aligned}
$$

As the matrix $\left(X^{2}-\frac{1}{3} \operatorname{Tr}\left(X^{2}\right) I\right)$ commutes with $e^{s X}$ and has vanishing trace, we can interprete $e^{s X}\left(X^{2}-\frac{1}{3} \operatorname{Tr}\left(X^{2}\right) I\right)$ as a tangent vector at the point $e^{s X}$. By decomposing the above expression into a tangent part and a part in the direction of the affine normal given by the position vector, we deduce that

$$
h\left(\gamma^{\prime}(s), \gamma^{\prime}(s)\right)=\frac{1}{3} \operatorname{Tr}\left(X^{2}\right) .
$$

So we see that $s$ is a constant length parametrisation of the curve $\gamma$ and therefore we have that $h\left(\gamma^{\prime}, \hat{\nabla}_{\gamma^{\prime}} \gamma^{\prime}\right)=0$ and

$$
h\left(\gamma^{\prime}, \nabla_{\gamma^{\prime}} \gamma^{\prime}\right)=h\left(\gamma^{\prime}, K\left(\gamma^{\prime}, \gamma^{\prime}\right)\right) \text {. }
$$

As

$$
\begin{aligned}
\gamma^{\prime \prime \prime}(s) & =\nabla_{\gamma^{\prime}(s)} \nabla_{\gamma^{\prime}(s)} \gamma^{\prime}(s)+h\left(\gamma^{\prime}, \gamma^{\prime}\right) \gamma^{\prime}+h\left(\gamma^{\prime}, K\left(\gamma^{\prime}, \gamma^{\prime}\right) \gamma\right. \\
& =e^{s X} X^{3} \\
& =e^{s X}\left(X^{3}-\frac{1}{3} \operatorname{Tr}\left(X^{3}\right) I\right) e^{s X^{T}}+\frac{1}{3} \operatorname{Tr}\left(X^{3}\right) \gamma(s),
\end{aligned}
$$

working at $s=0$ and writing $v=X$ as tangent vector, we have that

$$
\begin{aligned}
& h(v, v)=\frac{1}{3} \operatorname{Tr}\left(X^{2}\right), \\
& h(v, K(v, v))=\frac{1}{3} \operatorname{Tr} X^{3} .
\end{aligned}
$$

Linearising the above expressions, i.e. writing $v=\alpha_{1} v_{1}+\alpha_{2} v_{2}$, respectively $v=$ $\alpha_{1} v_{1}+\alpha_{2} v_{2}+\alpha_{3} v_{3}$, for $v_{i}=X_{i}, i=1,2,3$, and looking at the coefficient of $\alpha_{1} \alpha_{2}$, respectively $\alpha_{1} \alpha_{2} \alpha_{3}$ we obtain that

$$
\begin{aligned}
h\left(v_{1}, v_{2}\right) & =\frac{1}{3} \operatorname{Tr}\left(X_{1} X_{2}\right)=\frac{1}{3} \operatorname{Tr}\left(X_{2} X_{1}\right), \\
6 h\left(K\left(v_{1}, v_{2}\right), v_{3}\right) & =\frac{1}{3}\left(\operatorname{Tr} X_{1} X_{2} X_{3}+\operatorname{Tr} X_{3} X_{1} X_{2}+\operatorname{Tr} X_{2} X_{3} X_{1}+\operatorname{Tr} X_{1} X_{3} X_{2}+\operatorname{Tr} X_{3} X_{2} X_{1}+\operatorname{Tr} X_{2} X_{1} X_{3}\right) \\
& =\left(\operatorname{Tr} X_{1} X_{2} X_{3}+\operatorname{Tr} X_{2} X_{1} X_{3}\right) .
\end{aligned}
$$

So we see that

$$
K\left(v_{1}, v_{2}\right)=\frac{1}{2}\left(X_{1} X_{2}+X_{2} X_{1}-\frac{2}{3} \operatorname{Tr}\left(X_{1} X_{2}\right) I\right) .
$$

Indeed, we have that $\left(X_{1} X_{2}+X_{2} X_{1}-\frac{2}{3} \operatorname{Tr}\left(X_{1}, X_{2}\right) I\right)$ has vanishing trace and therefore $K\left(v_{1}, v_{2}\right)$ is indeed the unique tangent vector such that

$$
h\left(K\left(v_{1}, v_{2}\right), v_{3}\right)=\frac{1}{6}\left(\operatorname{Tr}\left(X_{1} X_{2} X_{3}\right)+\operatorname{Tr}\left(X_{2} X_{1} X_{3}\right)\right) .
$$

As by Cayley Hamilton for a matrix $X$ with vanishing trace we have that $X^{3}=$ $1 / 2 \operatorname{Tr}\left(X^{2}\right) X+\operatorname{det}(X) I$, we deduce that

$$
\operatorname{Tr} X^{4}=\frac{1}{2}\left(\operatorname{Tr} X^{2}\right)^{2},
$$


and therefore we have that

$$
\begin{aligned}
h(K(v, v), K(v, v)) & =\frac{1}{3} \operatorname{Tr}\left(X^{2}-\frac{1}{3} \operatorname{Tr} X^{2} I\right)^{2} \\
& =\frac{1}{3}\left(\operatorname{Tr} X^{4}+\frac{1}{9}\left(\operatorname{Tr} X^{2}\right)^{2} \operatorname{Tr} I-\frac{2}{3}\left(\operatorname{Tr} X^{2}\right)^{2}\right) \\
& =\frac{1}{18}\left(\operatorname{Tr} X^{2}\right)^{2} \\
& =\frac{1}{2}(h(v, v))^{2} .
\end{aligned}
$$

Hence $M_{1}$ is isotropic with positive $\lambda$. A straightforward computation also shows that the index of the metric is 3 .

Combining therefore the results in this section with Proposition 16 and the classification result of O. Birembaux and M. Djoric, see [2] in the positive definite case, we get the following theorem:

Theorem 18. Let $M$ be a 8-dimensional $\lambda$-isotropic affine hypersphere, with $\lambda \neq 0$. Therefore if necessary by changing the sign of the affine normal we may assume that $\lambda$ is positive. Then either

(1) the metric is positive definite, $M$ is isometric with $\frac{S L(3, \mathbb{C})}{S U(3)}$ and $M$ is affine congruent to an open part of the hypersurface $\left\{g \bar{g}^{T} \mid g \in S L(3, \mathbb{C})\right\}$ of $\mathbb{R}^{9}$, identified with the space of Hermitian symmetric matrices, or

(2) the metric has signature $4, M$ is isometric with $\frac{S L(3, \mathbb{C})}{S U(2,1)}$ and $M$ is affine congruent to an open part of the hypersurface $\left\{g A\left|g^{T}\right| g \in S L(3, \mathbb{C})\right\}$ of $\mathbb{R}^{9}$, where $A=\left(\begin{array}{ccc}1 & 0 & 0 \\ 0 & -1 & 0 \\ 0 & 0 & -1\end{array}\right)$, identified with the space of Hermitian symmetric matrices, or

(3) the metric has signature $3, M$ is isometric with $S L(3, \mathbb{R})$ and $M$ is affine congruent with $S L(3, \mathbb{R})$ considered as a hypersurface in $\mathbb{R}^{9}$ identified with $\mathbb{R}^{3 \times 3}$.

\section{AfFine hyperspheres of DIMENSION 14}

7.1. The form of $L, \operatorname{dim} \mathcal{U}^{\mathbb{C}}=4$.

We start with $w_{1} \in \mathcal{W}$ a vector with length 1 . As $L\left(w_{1}+i T w_{1}, w_{1}-i T w_{1}\right)$ is a real vector in $U$ with length $3 \mu^{2}$ there exists a real unit length vector $u_{1}$ in $U$ such that

$$
L\left(w_{1}+i T w_{1}, w_{1}-i T w_{1}\right)=\sqrt{3} \mu u_{1} .
$$

We now complete $u_{1}$ to a basis of $\mathcal{U}$ by choosing orthogonal $u_{2}, u_{3}, u_{4}$ in $\left\{u_{1}\right\}^{\perp}$ such that $h\left(u_{k}, u_{k}\right)=\varepsilon_{k}$, where $\varepsilon_{k}= \pm 1$. We also introduce $\delta_{k}$, for $\mathrm{k}=2,3,4$, by

$$
\delta_{k}=i, \quad \text { if } \varepsilon_{k}=-1 \quad \text { and } \quad \delta_{k}=1, \quad \text { if } \varepsilon_{k}=1 .
$$

Now we apply Proposition 11, which tells us that we can find vectors $w_{2}, w_{3}, w_{4}$ such that

$$
\begin{aligned}
& L\left(w_{1}+i T w_{1}, w_{2}-i T w_{2}\right)=\sqrt{3} \mu \delta_{2} u_{2}, \\
& L\left(w_{1}+i T w_{1}, w_{3}-i T w_{3}\right)=\sqrt{3} \mu \delta_{3} u_{3}, \\
& L\left(w_{1}+i T w_{1}, w_{4}-i T w_{4}\right)=\sqrt{3} \mu \delta_{4} u_{4} .
\end{aligned}
$$

The first two properties of Proposition 11 then tells us that $\left\{w_{1}, T w_{1}, \ldots, w_{4}, T w_{4}\right\}$ is a basis of $W$, as in Lemma 7 . Of course the previous equations also imply that

$$
L\left(w_{k}+i T w_{k}, w_{1}-i T w_{1}\right)=\sqrt{3} \mu \bar{\delta}_{k} u_{k} .
$$

We now look at $L\left(w_{2}+i T w_{2}, w_{3}-i T w_{3}\right)$. From the last part of Proposition 11 it follows that this vector is orthogonal to $L\left(w_{2}+i T w_{2}, w_{1}-i T w_{1}\right), L\left(w_{1}+\right.$ 
$\left.i T w_{1}, w_{3}-i T w_{3}\right)$ and $L\left(w_{1}+i T w_{1}, w_{1}-i T w_{1}\right)$. So this implies that there exists a complex number $b_{4}$ such that

$$
L\left(w_{2}+i T w_{2}, w_{3}-i T w_{3}\right)=b_{4} u_{4} .
$$

Similarly, we have that

$$
\begin{aligned}
& L\left(w_{2}+i T w_{2}, w_{4}-i T w_{4}\right)=b_{3} u_{3}, \\
& L\left(w_{3}+i T w_{3}, w_{4}-i T w_{4}\right)=b_{2} u_{2} .
\end{aligned}
$$

Using again Proposition 11 we see that there exists real numbers $c_{k}$ such that

$$
h\left(L\left(w_{k}+i T w_{k}, w_{k}-i T w_{k}\right), L\left(w_{k}+i T w_{k}, w_{k}-i T w_{k}\right)\right)=c_{k} u_{k} .
$$

From

(38) $h\left(L\left(w_{k}+i T w_{k}, w_{k}-i T w_{k}\right), L\left(w_{1}+i T w_{1}, w_{1}-i T w_{1}\right)\right)=$

$$
-h\left(L\left(w_{k}+i T w_{k}, w_{1}-i T w_{1}\right), L\left(w_{1}+i T w_{1}, w_{k}-i T w_{k}\right)\right),
$$

it follows that $c_{k}=-\sqrt{3} \mu \varepsilon_{k}$. Next we use the fact that for different indices $k$ and $\ell$ we have that

$$
\begin{aligned}
& h\left(L\left(w_{k}+i T w_{k}, w_{k}-i T w_{k}\right), L\left(w_{\ell}+i T w_{\ell}, w_{\ell}-i T w_{\ell}\right)\right)= \\
& -h\left(L\left(w_{k}+i T w_{k}, w_{\ell}-i T w_{\ell}\right), L\left(w_{\ell}+i T w_{\ell}, w_{k}-i T w_{k}\right)\right) .
\end{aligned}
$$

Expressing this for the different possibilities for $k$ and $\ell$ we find that

$$
\begin{aligned}
& 3 \mu^{2} \varepsilon_{2} \varepsilon_{3}=-\left|b_{4}\right|^{2} \varepsilon_{4} \\
& 3 \mu^{2} \varepsilon_{2} \varepsilon_{4}=-\left|b_{3}\right|^{2} \varepsilon_{3} \\
& 3 \mu^{2} \varepsilon_{4} \varepsilon_{3}=-\left|b_{2}\right|^{2} \varepsilon_{4} .
\end{aligned}
$$

Hence, up to permuting the vectors, we see that there are two possibilities. Either $\varepsilon_{2}=\varepsilon_{3}=\varepsilon_{4}=-1$, in which case the index of the metric is 8 or $\varepsilon_{2}=-1$ and $\varepsilon_{3}=\varepsilon_{4}=1$, in which case the index of the metric is 6 .

Computing the length of $L\left(w_{2}+i T w_{2}, w_{3}-i T w_{3}\right)$ we have in both cases that $b_{4}^{2} \varepsilon_{4}=3 \mu^{2}$. So if necessary by changing the sign of $u_{4}$ and $w_{4}$, we may assume that $b_{4}=\sqrt{3} \mu$.

We now complete the argument by looking at $h\left(L\left(w_{2}+i T w_{2}, w_{3}-i T w_{3}\right), L\left(w_{1}+i T w_{1}, w_{4}-i T w_{4}\right)=-h\left(L\left(w_{1}+i T w_{1}, w_{3}-i T w_{3}\right), L\left(w_{2}+i T w_{2}, w_{4}-i T w_{4}\right)\right.\right.$

This yields that $b_{3}=-\sqrt{3} \mu$. Interchanging the indices 2 and 3 in the formula above finally gives that $b_{2}=-\sqrt{3} \mu$ in the first case, and $-\sqrt{3} \mu i$ in the second case.

\subsection{Two canonical examples.}

First we look at the following example. We identify $\mathbb{R}^{15}$ with the set of all skew symmetric matrices in $\mathbb{R}^{6 \times 6}$. So an element $p \in \mathbb{R}^{15}$ is of the form

$$
p=\left(\begin{array}{cccccc}
0 & a_{1} & a_{2} & a_{3} & a_{4} & a_{5} \\
-a_{1} & 0 & a_{6} & a_{7} & a_{8} & a_{9} \\
-a_{2} & -a_{6} & 0 & a_{10} & a_{11} & a_{12} \\
-a_{3} & -a_{7} & -a_{10} & 0 & a_{13} & a_{14} \\
-a_{4} & -a_{8} & -a_{11} & -a_{13} & 0 & a_{15} \\
-a_{5} & -a_{9} & -a_{12} & -a_{14} & -a_{15} & 0
\end{array}\right)
$$

We take as hypersurface $M$ in $\mathbb{R}^{15}$ the skew symmetric matrices with determinant 1. Let $G=S L(6, \mathbb{R})$. Then, we have an action $\rho$ of $G$ on $M$ by $\rho(g)(p)=g p g^{T}$ 
Here we are interested in the connected component of the matrix

$$
I_{0}=\left(\begin{array}{cccccc}
0 & 0 & 0 & 0 & 0 & 1 \\
0 & 0 & 0 & 0 & 1 & 0 \\
0 & 0 & 0 & 1 & 0 & 0 \\
0 & 0 & -1 & 0 & 0 & 0 \\
0 & -1 & 0 & 0 & 0 & 0 \\
-1 & 0 & 0 & 0 & 0 & 0
\end{array}\right)
$$

If necessary, we restrict now $M$ to the orbit of $I_{0}$. Its isotropy group consists of the matrices $g$ of determinant 1 such that $g I_{0} g^{T}=I_{0}$. This Lie group is congruent to $S p(6)$ and therefore by Theorem 9.2 of [3] we know that $M_{1}$ is locally isometric with $\frac{S L(6, \mathbb{R})}{S p(6)}$.

Note that of course every element of $S L(6, \mathbb{R})$ acts at the same time also on $\mathbb{R}^{15}$ in a linear way and that therefore this action belongs to $G L(15, \mathbb{R})$. A straightforward computation shows that this action actually belongs to $S L(15, \mathbb{R})$. This implies that $M$ is at the same time an homogeneous affine hypersurface and by Proposition 6 an equiaffine sphere centered at the origin. So in order to determine the properties of $M$ it is sufficient to look at a single point.

In order to determine the tangent space at a point $p=g I_{0} g^{T}$, we look at the curves in $M$

$$
\gamma(s)=g e^{s X} I_{0} e^{s X^{T}} g^{T} .
$$

These are indeed curves in $M_{1}$, provided that $e^{s X} \in S L(6, \mathbb{R})$ or equivalently, provided that $\operatorname{Tr} X=0$. Note that $\gamma^{\prime}(s)=g e^{s X}\left(X I_{0}+I_{0} X^{T}\right) e^{s X^{T}} g^{T}$, where $v=\left(X I_{0}+I_{0} X^{T}\right)$ is a symmetric matrix. So by using a dimension argument we see that the tangent space is given by

$$
\left\{g v g^{T} \mid v=2 X I_{0}, X I_{0}=I_{0} X^{T}, \operatorname{Tr} X=0, X \in \mathbb{R}^{6 \times 6}\right\}=T_{p} M .
$$

In fact, such a matrix $X$ is of the form

$$
X=\left(\begin{array}{cccccc}
a_{1} & a_{2} & a_{3} & a_{4} & a_{5} & a_{6} \\
b_{1} & b_{2} & b_{3} & b_{4} & 0 & -a_{5} \\
c_{1} & c_{2} & -a_{1}-b_{2} & 0 & -b_{4} & -a_{4} \\
d_{1} & d_{2} & 0 & -a_{1}-b_{2} & b_{3} & a_{3} \\
e_{1} & 0 & -d_{2} & c_{2} & b_{2} & a_{2} \\
0 & -e_{1} & -d_{1} & c_{1} & b_{1} & a_{1}
\end{array}\right) .
$$

Working now at the point $I_{0}$, taking $g=I$ and $X \in\left\{X \in \mathbb{R}^{6 \times 6} \mid \operatorname{Tr} X=0, X I_{0}=\right.$ $\left.I_{0} X^{T}\right\}$ we see that

$$
\begin{aligned}
\nabla_{\gamma^{\prime}(s)} \gamma^{\prime}(s)+h\left(\gamma^{\prime}, \gamma^{\prime}\right) \gamma & =\gamma^{\prime \prime}(s) \\
& =e^{s X}\left(4 X^{2} I_{0}\right) e^{s X^{T}} \\
& =e^{s X}\left(\left(4 X^{2}-\frac{4}{6} \operatorname{Tr}\left(X^{2}\right) I\right) I_{0}\right) e^{s X^{T}}+\frac{4}{6} \operatorname{Tr}\left(X^{2}\right) e^{s X} I_{0} e^{s X^{T}} \\
& =e^{s X}\left(\left(4 X^{2}-\frac{4}{6} \operatorname{Tr}\left(X^{2}\right) I\right) I_{0}\right) e^{s X^{T}}+\frac{4}{6} \operatorname{Tr}\left(X^{2}\right) \gamma(s)
\end{aligned}
$$

As the matrix $\left(4 X^{2}-\frac{4}{6} \operatorname{Tr}\left(X^{2}\right) I\right)$ commutes with $I_{0}$, we can decompose the above expression into a tangent and a part in the direction of the affine normal given by the position vector, and therefore we find that

$$
h\left(\gamma^{\prime}(s), \gamma^{\prime}(s)\right)=\frac{4}{6} \operatorname{Tr}\left(X^{2}\right) .
$$

So we see that $s$ is a constant length parametrisation of the curve $\gamma$ and therefore we have that $h\left(\gamma^{\prime}, \hat{\nabla}_{\gamma^{\prime}} \gamma^{\prime}\right)=0$ and

$$
h\left(\gamma^{\prime}, \nabla_{\gamma^{\prime}} \gamma^{\prime}\right)=h\left(\gamma^{\prime}, K\left(\gamma^{\prime}, \gamma^{\prime}\right)\right)
$$


As

$$
\begin{aligned}
\gamma^{\prime \prime \prime}(s) & =\nabla_{\gamma^{\prime}(s)} \nabla_{\gamma^{\prime}(s)} \gamma^{\prime}(s)+h\left(\gamma^{\prime}, \gamma^{\prime}\right) \gamma^{\prime}+h\left(\gamma^{\prime}, K\left(\gamma^{\prime}, \gamma^{\prime}\right) \gamma\right. \\
& =e^{s X}\left(8 X^{3} I_{0}\right) e^{s X^{T}} \\
& =e^{s X}\left(\left(8 X^{3}-\frac{8}{6} \operatorname{Tr}\left(X^{3}\right) I\right) I_{0}\right) e^{s X^{T}}+\frac{8}{6} \operatorname{Tr}\left(X^{3}\right) \gamma(s),
\end{aligned}
$$

working at $s=0$ and writing $v=2 X I_{0}$ as tangent vector, we have that

$$
\begin{aligned}
& h(v, v)=\frac{4}{6} \operatorname{Tr}\left(X^{2}\right), \\
& h(v, K(v, v))=\frac{8}{6} \operatorname{Tr} X^{3} .
\end{aligned}
$$

Linearising the above expressions, i.e. writing $v=\alpha_{1} v_{1}+\alpha_{2} v_{2}$, respectively $v=$ $\alpha_{1} v_{1}+\alpha_{2} v_{2}+\alpha_{3} v_{3}$, for $v_{i}=2 X I_{0}, i=1,2,3$, and looking at the coefficient of $\alpha_{1} \alpha_{2}$, respectively $\alpha_{1} \alpha_{2} \alpha_{3}$ we obtain that

$$
\begin{aligned}
h\left(v_{1}, v_{2}\right) & =\frac{4}{6} \operatorname{Tr}\left(X_{1} X_{2}\right)=\frac{4}{6} \operatorname{Tr}\left(X_{2} X_{1}\right), \\
6 h\left(K\left(v_{1}, v_{2}\right), v_{3}\right) & =\frac{8}{3}\left(\operatorname{Tr} X_{1} X_{2} X_{3}+\operatorname{Tr} X_{3} X_{1} X_{2}+\operatorname{Tr} X_{2} X_{3} X_{1}+\operatorname{Tr} X_{1} X_{3} X_{2}+\operatorname{Tr} X_{3} X_{2} X_{1}+\operatorname{Tr} X_{2} X_{1} X_{3}\right) \\
& =4\left(\operatorname{Tr} X_{1} X_{2} X_{3}+\operatorname{Tr} X_{2} X_{1} X_{3}\right) .
\end{aligned}
$$

So we see that

$$
K\left(v_{1}, v_{2}\right)=2\left(X_{1} X_{2}+X_{2} X_{1}-\frac{2}{6} \operatorname{Tr}\left(X_{1} X_{2}\right) I\right) I_{0} .
$$

Indeed we have that $\left(X_{1} X_{2}+X_{2} X_{1}-\frac{2}{6} \operatorname{Tr}\left(X_{1} X_{2}\right) I\right)$ has vanishing trace, commutes with $I_{0}$ and therefore $K\left(v_{1}, v_{2}\right)$ is indeed the unique tangent vector such that

$$
h\left(K\left(v_{1}, v_{2}\right), v_{3}\right)=\frac{2}{3}\left(\operatorname{Tr}\left(X_{1} X_{2} X_{3}\right)+\operatorname{Tr}\left(X_{2} X_{1} X_{3}\right)\right) .
$$

By straightforward computations we deduce that

$$
\operatorname{Tr} X^{4}=\frac{1}{4}\left(\operatorname{Tr} X^{2}\right)^{2},
$$

and therefore we have that

$$
\begin{aligned}
h(K(v, v), K(v, v)) & =\frac{4}{6} \operatorname{Tr}\left(2 X^{2}-\frac{2}{3} \operatorname{Tr} X^{2} I\right)^{2} \\
& =\frac{4}{6}\left(4 \operatorname{Tr} X^{4}+\frac{4}{9}\left(\operatorname{Tr} X^{2}\right)^{2} \operatorname{Tr} I-\frac{8}{3}\left(\operatorname{Tr} X^{2}\right)^{2}\right) \\
& =\frac{2}{9}\left(\operatorname{Tr} X^{2}\right)^{2} \\
& =\frac{1}{2}(h(v, v))^{2} .
\end{aligned}
$$

Hence $M_{1}$ is isotropic with positive $\lambda$. A straightforward computation also shows that the index of the metric is 4 .

Next, the following example ilustrates the case when the signature of the indefinite metric on $M^{14}$ is 8 . First we identify $\mathbb{R}^{15}$ with the set of matrices $a=$ $\left\{\left(\begin{array}{cc}E & F \\ -\bar{F} & \bar{E}\end{array}\right), E=\bar{E}^{T}, F=-F^{T}\right\} \subset \mathbb{C}^{6 \times 6}$. An element in $a$ is of the form

$p:=\left(\begin{array}{cccccc}a_{1} & a_{2}+i a_{3} & a_{4}+i a_{5} & 0 & a_{6}+i a_{7} & a_{8}+i a_{9} \\ a_{2}-i a_{3} & a_{10} & a_{11}+i a_{12} & -a_{6}-i a_{7} & 0 & a_{13}+i a_{14} \\ a_{4}-i a_{5} & a_{11}+i a_{12} & a_{15} & -a_{8}-i a_{9} & -a_{13}-i a_{14} & 0 \\ 0 & -a_{6}+i a_{7} & -a_{8}+i a_{9} & a_{1} & a_{2}-i a_{3} & a_{4}-i a_{5} \\ a_{6}-i a_{7} & 0 & -a_{13}+i a_{14} & a_{2}+i a_{3} & a_{10} & a_{11}-i a_{12} \\ a_{8}-i a_{9} & a_{13}-i a_{14} & 0 & a_{4}+i a_{5} & a_{11}+i a_{12} & a_{15}\end{array}\right)$.

We take as hypersurface $M$ in $\mathbb{R}^{15}$ all such matrices with determinant 1 . Let $G=S U^{*}(6)$. Then, we have an action $\rho$ of $G$ on $M$ by $\rho(g)(p)=g p \bar{g}^{T}$. Note that $M$ has two connected components and that the action is transitive on each of the connected components. The connected component of $I$ has been studied in [2], 
where it was shown that it gives a positive definite isotropic affine hypersurface. Here we are interested in the connected component $M_{1}$ containing the matrix

$$
I_{0}=\left(\begin{array}{cccccc}
1 & 0 & 0 & 0 & 0 & 0 \\
0 & 1 & 0 & 0 & 0 & 0 \\
0 & 0 & -1 & 0 & 0 & 0 \\
0 & 0 & 0 & 1 & 0 & 0 \\
0 & 0 & 0 & 0 & 1 & 0 \\
0 & 0 & 0 & 0 & 0 & -1
\end{array}\right)
$$

Its isotropy group consists of the matrices $g$ of determinant 1 such that $g I_{0} \bar{g}^{T}=I_{0}$. This Lie group is congruent to $S p(1,2)$ and therefore, by Theorem 9.2 of [3], we know that $M$ is locally isometric with $\frac{S U^{*}(6)}{S p(1,2)}$.

Note that of course every element of $S U^{*}(6)$ acts at the same time also on $\mathbb{R}^{15}$ in a linear way and that therefore this action belongs to $G L(15, \mathbb{R})$. A straightforward computation shows that this action actually belongs to $S L(15, \mathbb{R})$. This implies that $M$ is at the same time an homogeneous affine hypersurface and, by Proposition 6 , an equiaffine sphere centered at the origin. So in order to determine the properties of $M_{1}$, it is sufficient to look at a single point.

In order to determine the tangent space at a point $p=g I_{0} \bar{g}^{T}$, we look at the curves in $M$

$$
\gamma(s)=g e^{s X} I_{0} e^{s \bar{X}^{T}} \bar{g}^{T} .
$$

These are indeed curves in $M_{1}$, provided that $\operatorname{Tr} X=0$ and $X J=J \bar{X}$, for $J=$ $\left(\begin{array}{cc}0 & I_{n} \\ -I_{n} & 0\end{array}\right)$. Note that $\gamma^{\prime}(s)=g e^{s X}\left(X I_{0}+I_{0} \bar{X}^{T}\right) e^{s \bar{X}^{T}} \bar{g}^{T}$. So by using a dimension argument, we see that the tangent space is given by

$$
\left\{g v \bar{g}^{T} \mid v=2 X I_{0}, X I_{0}=I_{0} \bar{X}^{T}, \operatorname{Tr} X=0, X J=J \bar{X}, X \in \mathbb{C}^{6 \times 6}\right\}=T_{p} M_{1} .
$$

In fact, such an $X$ if of the form

$$
\left(\begin{array}{cccccc}
-x-x_{0} & x_{1}+i y_{1} & x_{2}+i y_{2} & 0 & x_{3}-i y_{3} & x_{4}-i y_{4} \\
x_{1}-i y_{1} & x & x_{5}+i y_{5} & -x_{3}+i y_{3} & 0 & -x_{6}+i y_{6} \\
-x_{2}+i y_{2} & -x_{5}+i y_{5} & x_{0} & x_{4}-i y_{4} & x_{6}-i y_{6} & 0 \\
0 & -x_{3}-i y_{3} & -x_{4}-i y_{4} & -x-x_{0} & x_{1}-i y_{1} & -x_{2}-i y_{2} \\
x_{3}+i y_{3} & 0 & -x_{6}-i y_{6} & x_{1}+i y_{1} & x & -x_{5}+i y_{5} \\
x_{4}+i y_{4} & x_{6}+i y_{6} & 0 & x_{2}+i y_{2} & x_{5}+i y_{5} & x_{0}
\end{array}\right)
$$

Working now at the point $I_{0}$, taking $g=I$ and $X \in \mathbb{C}^{6 \times 6}$ satisfying $X I_{0}=$ $I_{0} \bar{X}^{T}, \operatorname{Tr} X=0, X J=J \bar{X}$, we see that

$$
\begin{aligned}
\nabla_{\gamma^{\prime}(s)} \gamma^{\prime}(s)+h\left(\gamma^{\prime}, \gamma^{\prime}\right) \gamma & =\gamma^{\prime \prime}(s) \\
& =e^{s X}\left(4 X^{2} I_{0}\right) e^{s \bar{X}^{T}} \\
& =e^{s X}\left(\left(4 X^{2}-\frac{4}{6} \operatorname{Tr}\left(X^{2}\right) I\right) I_{0}\right) e^{s \bar{X}^{T}}+\frac{4}{6} \operatorname{Tr}\left(X^{2}\right) e^{s X} I_{0} e^{s \bar{X}^{T}} \\
& =e^{s X}\left(\left(4 X^{2}-\frac{4}{6} \operatorname{Tr}\left(X^{2}\right) I\right) I_{0}\right) e^{s \bar{X}^{T}}+\frac{4}{6} \operatorname{Tr}\left(X^{2}\right) \gamma(s)
\end{aligned}
$$

As the matrix $\left(4 X^{2}-\frac{4}{6} \operatorname{Tr}\left(X^{2}\right) I\right)$ has the same properties as $X$, we can decompose the above expression into a tangent part and a part in the direction of the affine normal given by the position vector, and therefore we find that

$$
h\left(\gamma^{\prime}(s), \gamma^{\prime}(s)\right)=\frac{4}{6} \operatorname{Tr}\left(X^{2}\right) .
$$

So we see that $s$ is a constant length parametrisation of the curve $\gamma$ and therefore we have that $h\left(\gamma^{\prime}, \hat{\nabla}_{\gamma^{\prime}} \gamma^{\prime}\right)=0$ and

$$
h\left(\gamma^{\prime}, \nabla_{\gamma^{\prime}} \gamma^{\prime}\right)=h\left(\gamma^{\prime}, K\left(\gamma^{\prime}, \gamma^{\prime}\right)\right)
$$


As

$$
\begin{aligned}
\gamma^{\prime \prime \prime}(s) & =\nabla_{\gamma^{\prime}(s)} \nabla_{\gamma^{\prime}(s)} \gamma^{\prime}(s)+h\left(\gamma^{\prime}, \gamma^{\prime}\right) \gamma^{\prime}+h\left(\gamma^{\prime}, K\left(\gamma^{\prime}, \gamma^{\prime}\right) \gamma\right. \\
& =e^{s X}\left(8 X^{3} I_{0}\right) e^{s \bar{X}^{T}} \\
& =e^{s X}\left(\left(8 X^{3}-\frac{8}{6} \operatorname{Tr}\left(X^{3}\right) I\right) I_{0}\right) e^{s \bar{X}^{T}}+\frac{8}{6} \operatorname{Tr}\left(X^{3}\right) \gamma(s),
\end{aligned}
$$

working at $s=0$ and writing $v=2 X I_{0}$ as tangent vector, we have that

$$
\begin{aligned}
& h(v, v)=\frac{4}{6} \operatorname{Tr}\left(X^{2}\right), \\
& h(v, K(v, v))=\frac{8}{6} \operatorname{Tr} X^{3} .
\end{aligned}
$$

Linearising the above expressions, i.e. writing $v=\alpha_{1} v_{1}+\alpha_{2} v_{2}$, respectively $v=$ $\alpha_{1} v_{1}+\alpha_{2} v_{2}+\alpha_{3} v_{3}$, for $v_{i}=2 X I_{0}, i=1,2,3$, and looking at the coefficient of $\alpha_{1} \alpha_{2}$, respectively $\alpha_{1} \alpha_{2} \alpha_{3}$ we obtain that

$$
\begin{aligned}
h\left(v_{1}, v_{2}\right) & =\frac{4}{6} \operatorname{Tr}\left(X_{1} X_{2}\right)=\frac{4}{6} \operatorname{Tr}\left(X_{2} X_{1}\right), \\
6 h\left(K\left(v_{1}, v_{2}\right), v_{3}\right) & =\frac{8}{3}\left(\operatorname{Tr} X_{1} X_{2} X_{3}+\operatorname{Tr} X_{3} X_{1} X_{2}+\operatorname{Tr} X_{2} X_{3} X_{1}+\operatorname{Tr} X_{1} X_{3} X_{2}+\operatorname{Tr} X_{3} X_{2} X_{1}+\operatorname{Tr} X_{2} X_{1} X_{3}\right) \\
& =4\left(\operatorname{Tr} X_{1} X_{2} X_{3}+\operatorname{Tr} X_{2} X_{1} X_{3}\right) .
\end{aligned}
$$

So we see that

$$
K\left(v_{1}, v_{2}\right)=2\left(X_{1} X_{2}+X_{2} X_{1}-\frac{2}{6} \operatorname{Tr}\left(X_{1} X_{2}\right) I\right) I_{0} .
$$

Indeed we have that $\left(X_{1} X_{2}+X_{2} X_{1}-\frac{2}{6} \operatorname{Tr}\left(X_{1} X_{2}\right) I\right)$ has vanishing trace, commutes with $I_{0}$ and therefore $K\left(v_{1}, v_{2}\right)$ is indeed the unique tangent vector such that

$$
h\left(K\left(v_{1}, v_{2}\right), v_{3}\right)=\frac{2}{3}\left(\operatorname{Tr}\left(X_{1} X_{2} X_{3}\right)+\operatorname{Tr}\left(X_{2} X_{1} X_{3}\right)\right) .
$$

By straightforward computations we deduce that

$$
\operatorname{Tr} X^{4}=\frac{1}{4}\left(\operatorname{Tr} X^{2}\right)^{2}
$$

and therefore we have that

$$
\begin{aligned}
h(K(v, v), K(v, v)) & =\frac{4}{6} \operatorname{Tr}\left(2 X^{2}-\frac{2}{3} \operatorname{Tr} X^{2} I\right)^{2} \\
& =\frac{4}{6}\left(4 \operatorname{Tr} X^{4}+\frac{4}{9}\left(\operatorname{Tr} X^{2}\right)^{2} \operatorname{Tr} I-\frac{8}{3}\left(\operatorname{Tr} X^{2}\right)^{2}\right) \\
& =\frac{2}{9}\left(\operatorname{Tr} X^{2}\right)^{2} \\
& =\frac{1}{2}(h(v, v))^{2} .
\end{aligned}
$$

Hence $M_{1}$ is isotropic with positive $\lambda$. A straightforward computation also shows that the index of the metric is 8 .

\section{AfFine hyperspheres of Dimension 26}

8.1. The form of $L, \operatorname{dim} \mathcal{U}=8$.

Before treating each case of the signature for the metric, we first will give some lemmas which will be very useful in order to simplify the proof significantly. We start with an arbitrary vector $w+i T w \in \mathcal{W}_{1}$ with length 2 and define a real vector $\sqrt{3} \mu u=L(w+i T w, w-i T w)$. We call $w_{1}^{1}=w$ and $w_{2}^{1}=T w$. Next, we choose arbitrary orthogonal vectors $u_{2}, \ldots, u_{8}$ such that $u_{1}, u_{2}, \ldots, u_{8}$ forms an orthonormal (real) basis in $\mathcal{U}$, that is $h\left(u_{j}, u_{k}\right)=\varepsilon_{j} \delta_{j k}$, where $\varepsilon_{j}= \pm 1$ indicate the length of $u_{j}$. As the operator $L\left(\omega_{1}^{1}+i \omega_{2}^{1},-\right)$ is bijective, for every $u_{j}$ we find $\omega_{j}^{1}, \omega_{j}^{2}$, such that

$$
L\left(\omega_{1}^{1}+i \omega_{2}^{1}, \omega_{1}^{j}-i \omega_{2}^{j}\right)=\sqrt{3} \mu \delta_{j} u_{j}, \text { where } \delta_{j}=\left\{\begin{array}{l}
1, \text { if } \varepsilon=1 \\
i, \text { if } \varepsilon=-1 .
\end{array}\right.
$$

Lemma 19. For the previously defined vectors, $L$ satisfies

$$
L\left(\omega_{1}^{k}+i \omega_{2}^{k}, \omega_{1}^{k}-i \omega_{2}^{k}\right)=-\sqrt{3} \mu \varepsilon_{k} u_{1} .
$$


Proof. The result is straightforward, by properties (23) and (24):

(41)

$$
\begin{aligned}
h\left(L\left(\omega_{1}^{k}+i \omega_{2}^{k}, \omega_{1}^{k}-i \omega_{2}^{k}\right),\right. & \left.L\left(\omega_{1}^{1}+i \omega_{2}^{1}, \omega_{1}^{j}-i \omega_{2}^{j}\right)\right)= \\
& =-h\left(L\left(\omega_{1}^{1}+i \omega_{2}^{1}, \omega_{1}^{k}-i \omega_{2}^{k}\right), L\left(\omega_{1}^{k}+i \omega_{2}^{k}, \omega_{1}^{j}-i \omega_{2}^{j}\right)\right) \\
& =-\frac{\delta}{\bar{\delta}} h\left(L\left(\omega_{1}^{k}+i \omega_{2}^{k}, \omega_{1}^{1}-i \omega_{2}^{1}\right), L\left(\omega_{1}^{k}+i \omega_{2}^{k}, \omega_{1}^{j}-i \omega_{2}^{j}\right)\right) \\
& \left.=-\frac{\delta}{\bar{\delta}} \frac{3 \mu^{2}}{2} h\left(\omega_{1}^{1}+i \omega_{2}^{1}, \omega_{1}^{j}-i \omega_{2}^{j}\right)\right) \\
& =\left\{\begin{array}{l}
0, j \neq 1 \\
-3 \mu^{2} \varepsilon_{k}, \quad j=1 .
\end{array}\right.
\end{aligned}
$$

Lemma 20. Let $u_{j}$ and $u_{k}$ determine $\varepsilon_{j}$ and $\varepsilon_{k}$ such that $\varepsilon_{j}=\varepsilon_{k}$, for $k, j>1$. Then $L\left(\omega_{1}^{k}+i \omega_{2}^{k}, \omega_{1}^{j}-i \omega_{2}^{j}\right)$ is an imaginary vector.

Proof. Let us define the orthonormal basis of $\mathcal{U}$ given by

$$
\left\{\begin{array}{l}
u_{k}^{*}=\cos (t) u_{k}+\sin (t) u_{j}, \\
u_{j}^{*}=-\sin (t) u_{k}+\cos (t) u_{k}, \\
u_{l}^{*}=u_{l}, l \neq k, j .
\end{array}\right.
$$

By relation (40), we compute

$$
L\left(\omega_{1}^{1}+i \omega_{2}^{1}, \cos (t)\left(\omega_{1}^{k}-i \omega_{2}^{k}\right)+\sin (t)\left(\omega_{1}^{j}-i \omega_{2}^{j}\right)\right)=\sqrt{3} \mu \delta_{j}\left(\cos (t) u_{k}+\sin (t) u_{j}\right)
$$

and therefore we find $\omega_{1}^{* k}+i \omega_{2}^{* k}=\cos (t)\left(\omega_{1}^{k}+i \omega_{2}^{k}\right)+\sin (t)\left(\omega_{1}^{j}+i \omega_{2}^{j}\right)$ and $\omega_{1}^{* j}+i \omega_{2}^{* j}=$ $-\sin (t)\left(\omega_{1}^{k}+i \omega_{2}^{k}\right)+\cos (t)\left(\omega_{1}^{j}+i \omega_{2}^{j}\right)$ such that

$$
L\left(\omega_{1}^{1}+i \omega_{2}^{1}, \omega_{1}^{* k}+i \omega_{2}^{* k}\right)=\sqrt{3} \delta_{k} u_{k}^{*} .
$$

Next, by lemma (19) we may write

$$
L\left(\omega_{1}^{* k}+i \omega_{2}^{* k}, \omega_{1}^{* k}-i \omega_{2}^{* k}\right)=-\sqrt{3} \mu \varepsilon_{k} u_{1}
$$

and using the bilinearity of $L$, we get the conclusion.

Lemma 21. Let $u_{j}$ and $u_{k}$ determine $\varepsilon_{j}$ and $\varepsilon_{k}$ such that $\varepsilon_{j}=-1$ and $\varepsilon_{k}=1$, for $k, j>1$. Then $L\left(\omega_{1}^{k}+i \omega_{2}^{k}, \omega_{1}^{j}-i \omega_{2}^{j}\right)$ is a real vector.

Proof. First, define an orthonormal basis of $\mathcal{U}$ given by

$$
\left\{\begin{array}{l}
u_{k}^{*}=\cosh (t) u_{k}+\sinh (t) u_{j}, \\
u_{j}^{*}=\sinh (t) u_{k}+\cosh (t) u_{k} \\
u *_{l}=u_{l}, l \neq k, j
\end{array}\right.
$$

and notice that $L\left(\omega_{1}^{1}+i \omega_{2}^{1}, \omega_{1}^{k}-i \omega_{2}^{k}\right)=\sqrt{3} \mu u_{k}$ and $L\left(\omega_{1}^{1}+i \omega_{2}^{1}, \omega_{1}^{j}-i \omega_{2}^{j}\right) \sqrt{3} \mu u_{j}$. We take $a, b, c, d$ complex functions and find $\omega_{1}^{* k}-i \omega_{2}^{* k}=a\left(\omega_{1}^{k}-i \omega_{2}^{k}\right)+b\left(\omega_{1}^{j}-i \omega_{2}^{j}\right)$ and $\omega_{1}^{* j}-i \omega_{2}^{* j}=c\left(\omega_{1}^{k}-i \omega_{2}^{k}\right)+d\left(\omega_{1}^{j}-i \omega_{2}^{j}\right)$ to be the unique vectors satisfying

$$
L\left(\omega_{1}^{1}+i \omega_{2}^{1}, \omega_{1}^{* k}-i \omega_{2}^{* k}\right)=\sqrt{3} \mu u_{k}^{*} \quad \text { and } \quad L\left(\omega_{1}^{1}+i \omega_{2}^{1}, \omega_{1}^{* j}-i \omega_{2}^{* j}\right) \sqrt{3} \mu u_{j}^{*} .
$$

Therefore, we find

$$
a=\cosh (t), \quad b=i \sinh (t), \quad c=-i \sinh (t) \quad \text { and } \quad d=\cosh (t) .
$$

Finally, using the bilinearity of $L$, the conclusion follows easily from

$$
L\left(\omega_{1}^{* k}+i \omega_{2}^{* k}, \omega_{1}^{* k}-i \omega_{2}^{* k}\right)=-\sqrt{3} \mu u_{1} .
$$


In what follows, we will study the remaining cases for the metric on $\mathcal{U}$. First we deal with the case that the signature of the metric is 4,5 or 6 . Let $u_{1}$ be defined as in the beginning of this section. Next, choose $u_{2} \perp u_{1}$ such that $h\left(u_{2}, u_{2}\right)=-1$ and $w_{1}^{2}$ and $w_{2}^{2}$ such that $L\left(\omega_{1}^{1}+i \omega_{2}^{1}, \omega_{1}^{2}-i \omega_{2}^{2}\right)=\sqrt{3} \mu u_{2}$ and $u_{3} \perp u_{1}, u_{2}$ and $w_{1}^{3}$ and $w_{2}^{3}$ such that $h\left(u_{3}, u_{3}\right)=-1, L\left(\omega_{1}^{1}+i \omega_{2}^{1}, \omega_{1}^{3}-i \omega_{2}^{3}\right)=\sqrt{3} \mu u_{3}$. Then, we look at the vector $L\left(\omega_{1}^{2}+i \omega_{2}^{2}, \omega_{1}^{3}-i \omega_{2}^{3}\right)$ and see, by property (23), that it is orthogonal to $u_{1}, u_{2}$ and $u_{3}$ and has length $3 \mu^{2}$ and by lemma (20), that it is an imaginary vector. Therefore, we define $u_{4}$ of length -1 such that

$$
L\left(\omega_{1}^{2}+i \omega_{2}^{2}, \omega_{1}^{3}-i \omega_{2}^{3}\right)=\sqrt{3} \mu i u_{4} .
$$

Next, by surjectivity of $L\left(\omega_{1}^{1}+i \omega_{2}^{1},-\right)$ and by (40) we can pick $w_{1}^{4}$ and $w_{2}^{4}$ such that $L\left(\omega_{1}^{1}+i \omega_{2}^{1}, \omega_{1}^{4}-i \omega_{2}^{4}\right)=\sqrt{3} \mu i u_{4}$. In the following, we pick $u_{5} \perp u_{1}, u_{2}, u_{3}, u_{4}$ of length 1 and obtain $\omega_{1}^{5}, \omega_{2}^{5}$ such that $L\left(\omega_{1}^{1}+i \omega_{2}^{1}, \omega_{1}^{5}-i \omega_{2}^{5}\right)=\sqrt{3} \mu u_{5}$. Remark that the vectors $L\left(\omega_{1}^{2}+i \omega_{2}^{2}, \omega_{1}^{5}-i \omega_{2}^{5}\right), L\left(\omega_{1}^{3}+i \omega_{2}^{3}, \omega_{1}^{5}-i \omega_{2}^{5}\right), L\left(\omega_{1}^{4}+i \omega_{2}^{4}, \omega_{1}^{5}-i \omega_{2}^{5}\right)$ are real, of positive length, mutually orthogonal and orthogonal to $u_{1}, u_{5}$. Therefore, the choice of $u_{1}, \ldots, u_{5}$ implies that the metric on $\left\{u_{2}, u_{3}, u_{4}\right\}^{\perp}$ is positive definite. Therefore, the cases when the metric has signature 4,5 or 6 cannot happen.

In case that the index is 0 , we proceed as follows. Let $u_{1}$ be defined as before, choose $u_{2} \perp u_{1}$ of length 1 and obtain the existence of $\omega_{1}^{2}, \omega_{2}^{2}$ such that $L\left(\omega_{1}^{1}+\right.$ $\left.i \omega_{2}^{1}, \omega_{1}^{2}-i \omega_{2}^{2}\right)=\sqrt{3} \mu u_{2}$ and $L\left(\omega_{1}^{2}+i \omega_{2}^{2}, \omega_{1}^{2}-i \omega_{2}^{2}\right)=-\sqrt{3} \mu u_{1}$. Then, choose $u_{3} \perp u_{1}, u_{2}$ of length 1 and obtain again $L\left(\omega_{1}^{1}+i \omega_{2}^{1}, \omega_{1}^{3}-i \omega_{2}^{3}\right)=\sqrt{3} \mu u_{3}$ and $L\left(\omega_{1}^{3}+i \omega_{2}^{3}, \omega_{1}^{3}-i \omega_{2}^{3}\right)=-\sqrt{3} \mu u_{1}$. Moreover, the vector $L\left(\omega_{1}^{2}+i \omega_{2}^{2}, \omega_{1}^{3}-i \omega_{2}^{3}\right)$ is an imaginary vector, orthogonal on $u_{1}, u_{2}, u_{3}$ (by relation (23)) and therefore, we get the existence of a unit vector of negative length, $u_{4}$, such that $L\left(\omega_{1}^{2}+i \omega_{2}^{2}, \omega_{1}^{3}-i \omega_{2}^{3}\right)=$ $\sqrt{3} \mu i u_{4}$. This contradicts the fact that the index equals 0 .

Next, we start anew, with different choices of vectors in order to eliminate the case when the signature of the metric is 1 .

Let $u_{1}$ be defined as before, choose $u_{2} \perp u_{1}$ of length -1 and obtain the existence of $\omega_{1}^{2}, \omega_{2}^{2}$ such that $L\left(\omega_{1}^{1}+i \omega_{2}^{1}, \omega_{1}^{2}-i \omega_{2}^{2}\right)=\sqrt{3} \mu i u_{2}$ and $L\left(\omega_{1}^{2}+i \omega_{2}^{2}, \omega_{1}^{2}-i \omega_{2}^{2}\right)=\sqrt{3} \mu u_{1}$. Then, choose $u_{3} \perp u_{1}, u_{2}$ of length 1 and obtain again $L\left(\omega_{1}^{1}+i \omega_{2}^{1}, \omega_{1}^{3}-i \omega_{2}^{3}\right)=\sqrt{3} \mu u_{3}$ and $L\left(\omega_{1}^{3}+i \omega_{2}^{3}, \omega_{1}^{3}-i \omega_{2}^{3}\right)=-\sqrt{3} \mu u_{1}$. Moreover, the vector $L\left(\omega_{1}^{2}+i \omega_{2}^{2}, \omega_{1}^{3}-i \omega_{2}^{3}\right)$ is a real vector, orthogonal on $u_{1}, u_{2}, u_{3}$ (by relation (23)) and therefore, we get the existence of a unit vector of positive length, $u_{4}$, such that $L\left(\omega_{1}^{2}+i \omega_{2}^{2}, \omega_{1}^{3}-i \omega_{2}^{3}\right)=$ $\sqrt{3} \mu u_{4}$. Consequently, $L\left(\omega_{1}^{1}+i \omega_{2}^{1}, \omega_{1}^{4}-i \omega_{2}^{4}\right)=\sqrt{3} \mu u_{4}$ and $L\left(\omega_{1}^{4}+i \omega_{2}^{4}, \omega_{1}^{4}-i \omega_{2}^{4}\right)=$ $-\sqrt{3} \mu u_{1}$. Next, we pick $u_{5} \perp u_{1}, u_{2}, u_{3}, u_{4}$ of length 1 and find

$$
L\left(\omega_{1}^{1}+i \omega_{2}^{1}, \omega_{1}^{5}-i \omega_{2}^{5}\right)=\sqrt{3} \mu u_{5} \quad \text { and } \quad L\left(\omega_{1}^{5}+i \omega_{2}^{5}, \omega_{1}^{5}-i \omega_{2}^{5}\right)=-\sqrt{3} \mu u_{1} .
$$

Finally, by lemma (20) and the property in (23), we see that the vectors $L\left(\omega_{1}^{3}+\right.$ $\left.i \omega_{2}^{3}, \omega_{1}^{5}-i \omega_{2}^{5}\right)$ and $L\left(\omega_{1}^{3}+i \omega_{2}^{3}, \omega_{1}^{4}-i \omega_{2}^{4}\right)$ are orthogonal imaginary vectors. This implies that the index of the metric is at least 2 .

Now, we will prove that the metric on $U$ cannot have signature 2 . Let $u_{1}$ be defined as in (??), choose $u_{2} \perp u_{1}$ of length -1 and obtain $\omega_{1}^{2}, \omega_{2}^{2}$ such that $L\left(\omega_{1}^{1}+i \omega_{2}^{1}, \omega_{1}^{2}-i \omega_{2}^{2}\right)=\sqrt{3} \mu i u_{2}$ and $L\left(\omega_{1}^{2}+i \omega_{2}^{2}, \omega_{1}^{2}-i \omega_{2}^{2}\right)=\sqrt{3} \mu u_{1}$. Then, choose $u_{3} \perp u_{1}, u_{2}$ of length -1 and obtain again $L\left(\omega_{1}^{1}+i \omega_{2}^{1}, \omega_{1}^{3}-i \omega_{2}^{3}\right)=\sqrt{3} \mu i u_{3}$ and $L\left(\omega_{1}^{3}+i \omega_{2}^{3}, \omega_{1}^{3}-i \omega_{2}^{3}\right)=\sqrt{3} \mu u_{1}$. Remark now that the vector $L\left(\omega_{1}^{2}+i \omega_{2}^{2}, \omega_{1}^{3}-i \omega_{2}^{3}\right)$ is an imaginary vector, orthogonal on $u_{1}, u_{2}, u_{3}$ (by relation $(23)$ ). So we have that $L\left(\omega_{1}^{2}+i \omega_{2}^{2}, \omega_{1}^{3}-i \omega_{2}^{3}\right)=\sqrt{3} \mu i u_{4}$, where $u_{4}$ has negative length and belongs to $\left\{u_{1}, u_{2}, u_{3}\right\}^{\perp}$, where the metric is positive definite, which is a contradiction.

Next we deal with the case that the index of the metric equals 7 . So on $\left\{u_{1}\right\}^{\perp}$ the metric is negative definite. We may take $u_{2} \in \mathcal{U}$ such that $h\left(u_{2}, u_{2}\right)=-1$ and $h\left(u_{1}, u_{2}\right)=0$. 
As $L\left(\omega_{1}^{1}+i \omega_{2}^{1},-\right)$ is a surjective operator, we can pick $w_{1}^{1}$ and $w_{2}^{1}=T w_{1}^{1}$ such that

$$
\begin{gathered}
L\left(\omega_{1}^{1}+i \omega_{2}^{1}, \omega_{1}^{2}-i \omega_{2}^{2}\right)=\sqrt{3} \mu i u_{2}, \\
L\left(\omega_{1}^{2}+i \omega_{2}^{2}, \omega_{1}^{1}-i \omega_{2}^{1}\right)=-\sqrt{3} \mu i u_{2} .
\end{gathered}
$$

and by the lemma we have $L\left(\omega_{1}^{2}+i \omega_{2}^{2}, \omega_{1}^{2}-i \omega_{2}^{2}\right)=\sqrt{3} \mu u_{1}$ Next, we take $u_{3} \in \mathcal{U}$ such that $h\left(u_{3}, u_{3}\right)=-1$. In a similar way as before, we define $\omega_{1}^{3}$ and $\omega_{2}^{3}$ and obtain

$$
L\left(\omega_{1}^{1}+i \omega_{2}^{1}, \omega_{1}^{3}-i \omega_{2}^{3}\right)=\sqrt{3} \mu i u_{3} .
$$

By the lemma this implies that $L\left(\omega_{1}^{3}+i \omega_{2}^{3}, \omega_{1}^{3}-i \omega_{2}^{3}\right)=\sqrt{3} \mu u_{1}$. Next, we find that $L\left(\omega_{1}^{3}+i \omega_{2}^{3}, \omega_{1}^{2}-i \omega_{2}^{2}\right)$ is an imaginary vector which is orthogonal to $u_{1}, u_{2}$ and $u_{3}$ such that we may write $L\left(\omega_{1}^{3}+i \omega_{2}^{3}, \omega_{1}^{2}-i \omega_{2}^{2}\right)=\sqrt{3} \mu i u_{4}$, for some $u_{4} \in U, u_{4} \perp u_{1}, u_{2}, u_{3}$. Given $u_{4}$, we define new $\omega_{1}^{4}$ and $\omega_{2}^{4}$ in $\mathcal{W}_{2}$ such that $L\left(\omega_{1}^{1}+i \omega_{2}^{1}, \omega_{1}^{4}-i \omega_{2}^{4}\right)=$ $\sqrt{3} \mu i u_{4}$ and we have $L\left(\omega_{1}^{4}+i \omega_{2}^{4}, \omega_{1}^{4}-i \omega_{2}^{4}\right)=\sqrt{3} \mu u_{1}$. Next, we want to determine $L\left(\omega_{1}^{2}+i \omega_{2}^{2}, \omega_{1}^{4}-i \omega_{2}^{4}\right)$. We immediately obtain that it is an imaginary vector of length $3 \mu^{2}$ which is orthogonal to $u_{1}, u_{2}$ and $u_{4}$. As

$h\left(L\left(\omega_{1}^{2}+i \omega_{2}^{2}, \omega_{1}^{4}-i \omega_{2}^{4}\right), L\left(\omega_{1}^{1}+i \omega_{2}^{1}, \omega_{1}^{3}-i \omega_{2}^{3}\right)\right)=-h\left(L\left(\omega_{1}^{2}+i \omega_{2}^{2}, \omega_{1}^{3}-i \omega_{2}^{3}\right), L\left(\omega_{1}^{1}+i \omega_{2}^{1}, \omega_{1}^{4}-i \omega_{2}^{4}\right)\right)=3 \mu^{2}$,

it follows from the Cauchy-Schwartz inequality on $\left\{u_{1}\right\}^{\perp}$ that $L\left(\omega_{1}^{2}+i \omega_{2}^{2}, \omega_{1}^{4}-\right.$ $\left.i \omega_{2}^{4}\right)=\sqrt{3} \mu i u_{3}$. Similarly it follows that $L\left(\omega_{1}^{4}+i \omega_{2}^{4}, \omega_{1}^{3}-i \omega_{2}^{3}\right)==\sqrt{3} \mu i u_{2}$.

Remember that so far we have defined $u_{1}, u_{2}, u_{3}$ and $u_{4} \in \mathcal{U}$ and $\omega_{1}^{1}, \omega_{2}^{1}, \omega_{1}^{2}, \omega_{2}^{2}$, $\omega_{1}^{3}, \omega_{2}^{3}, \omega_{1}^{4}, \omega_{2}^{4} \in \mathcal{W}$. We take now some arbitrary $u_{5} \in\left\{u_{1}, u_{2}, u_{3}, u_{4}\right\}^{\perp}$ such that $h\left(u_{5}, u_{5}\right)=-1$ and use again the surjectivity of $L\left(\omega_{1}^{1}+i \omega_{2}^{1},-\right)$ to define $w_{1}^{5}$ and $w_{2}^{5}=T w_{1}^{5}$ such that $L\left(\omega_{1}^{1}+i \omega_{2}^{1}, \omega_{1}^{5}-i \omega_{2}^{5}\right)=\sqrt{3} \mu i u_{5}$ and

$$
L\left(\omega_{1}^{5}+i \omega_{2}^{5}, \omega_{1}^{5}-i \omega_{2}^{5}\right)=\sqrt{3} \mu u_{1} .
$$

Next, we proceed with the computations as we did, for instance, for $L\left(\omega_{1}^{3}+i \omega_{2}^{3}, \omega_{1}^{2}-\right.$ $\left.i \omega_{2}^{2}\right)$ and define $u_{6}, u_{7}, u_{8} \in \mathcal{U}$ such that

$$
\begin{aligned}
& L\left(\omega_{1}^{5}+i \omega_{2}^{5}, \omega_{1}^{2}-i \omega_{2}^{2}\right)=\sqrt{3} \mu i u_{6}, \\
& L\left(\omega_{1}^{5}+i \omega_{2}^{5}, \omega_{1}^{3}-i \omega_{2}^{3}\right)=\sqrt{3} \mu i u_{7}, \\
& L\left(\omega_{1}^{5}+i \omega_{2}^{5}, \omega_{1}^{4}-i \omega_{2}^{4}\right)=\sqrt{3} \mu i u_{8} .
\end{aligned}
$$

Given $u_{6}, u_{7}, u_{8}$, we use the surjectivity of $L\left(\omega_{1}^{1}+i \omega_{2}^{1},-\right)$ and just like previously done, we define $\omega_{1}^{k}, \omega_{2}^{k} \in \mathcal{U}$, for $k=6,7,8$ and determine

$$
L\left(\omega_{1}^{1}+i \omega_{2}^{1}, \omega_{1}^{k}-i \omega_{2}^{k}\right)=\sqrt{3} \mu i u_{k} .
$$

Next, we find $L\left(\omega_{1}^{k}+i \omega_{2}^{k}, \omega_{1}^{k}-i \omega_{2}^{k}\right)=\sqrt{3} \mu u_{1}$ for $k=6,7,8$. Then, we compute similarly as for $L\left(\omega_{1}^{2}+i \omega_{2}^{2}, \omega_{1}^{4}-i \omega_{2}^{4}\right)$ in order to determine

$$
L\left(\omega_{1}^{6}+i \omega_{2}^{6}, \omega_{1}^{2}-i \omega_{2}^{2}\right)=-\sqrt{3} \mu i u_{5} .
$$

As for the vectors $L\left(\omega_{1}^{3}+i \omega_{2}^{3}, \omega_{1}^{6}-i \omega_{2}^{6}\right), L\left(\omega_{1}^{4}+i \omega_{2}^{4}, \omega_{1}^{6}-i \omega_{2}^{6}\right)$ and $L\left(\omega_{1}^{2}+i \omega_{2}^{2}, \omega_{1}^{7}-\right.$ $\left.i \omega_{2}^{7}\right)$, by using property (24) and the determined vectors so far, we see they are in the directions of $u_{8}, u_{7}$ and $u_{8}$ respectively. We can easily determine their components by following the same procedure as for $L\left(\omega_{1}^{3}+i \omega_{2}^{3}, \omega_{1}^{2}-i \omega_{2}^{2}\right)$. Thus, we may write

$$
\begin{aligned}
& L\left(\omega_{1}^{3}+i \omega_{2}^{3}, \omega_{1}^{6}-i \omega_{2}^{6}\right)=\varepsilon u_{8}, \\
& L\left(\omega_{1}^{4}+i \omega_{2}^{4}, \omega_{1}^{6}-i \omega_{2}^{6}\right)=\varepsilon_{1} u_{7}, \\
& L\left(\omega_{1}^{2}+i \omega_{2}^{2}, \omega_{1}^{7}-i \omega_{2}^{7}\right)=\varepsilon_{2} u_{8},
\end{aligned}
$$


where $\varepsilon, \varepsilon_{1}, \varepsilon_{2}= \pm \sqrt{3} \mu i$. Further on, in order to determine $L\left(\omega_{1}^{3}+i \omega_{2}^{3}, \omega_{1}^{7}-i \omega_{2}^{7}\right)$, we first see by property (24) that it is orthogonal to $\left\{u_{1}, u_{2}, u_{3}, u_{4}, u_{7}, u_{8}\right\}$. Next, as

$$
\begin{aligned}
& h\left(L\left(\omega_{1}^{3}+i \omega_{2}^{3}, \omega_{1}^{7}-i \omega_{2}^{7}\right), L\left(\omega_{1}^{1}+i \omega_{2}^{1}, \omega_{1}^{6}-i \omega_{2}^{6}\right)\right)+ \\
& h\left(L\left(\omega_{1}^{1}+i \omega_{2}^{1}, \omega_{1}^{7}-i \omega_{2}^{7}\right), L\left(\omega_{1}^{3}+i \omega_{2}^{3}, \omega_{1}^{6}-i \omega_{2}^{6}\right)\right)=0
\end{aligned}
$$

and

(48) $\quad h\left(L\left(\omega_{1}^{3}+i \omega_{2}^{3}, \omega_{1}^{7}-i \omega_{2}^{7}\right), L\left(\omega_{1}^{1}+i \omega_{2}^{1}, \omega_{1}^{5}-i \omega_{2}^{5}\right)\right)+$

$$
h\left(L\left(\omega_{1}^{1}+i \omega_{2}^{1}, \omega_{1}^{7}-i \omega_{2}^{7}\right), L\left(\omega_{1}^{3}+i \omega_{2}^{3}, \omega_{1}^{5}-i \omega_{2}^{5}\right)\right)=0
$$

we find

$$
L\left(\omega_{1}^{3}+i \omega_{2}^{3}, \omega_{1}^{7}-i \omega_{2}^{7}\right)=\sqrt{3} \mu i u_{5} .
$$

It is easy to see that $L\left(\omega_{1}^{4}+i \omega_{2}^{4}, \omega_{1}^{7}-i \omega_{2}^{7}\right)$ is coliniar with $u_{6}$. From (23) we obtain

$$
\begin{gathered}
h\left(L\left(\omega_{1}^{1}+i \omega_{2}^{1}, \omega_{1}^{6}-i \omega_{2}^{6}\right), L\left(\omega_{1}^{4}+i \omega_{2}^{4}, \omega_{1}^{7}-i \omega_{2}^{7}\right)\right)+ \\
h\left(L\left(\omega_{1}^{4}+i \omega_{2}^{4}, \omega_{1}^{6}-i \omega_{2}^{6}\right), L\left(\omega_{1}^{1}+i \omega_{2}^{1}, \omega_{1}^{7}-i \omega_{2}^{7}\right)\right)=0 \Leftrightarrow \\
h\left(u_{6}, L\left(\omega_{1}^{4}+i \omega_{2}^{4}, \omega_{1}^{7}-i \omega_{2}^{7}\right)\right)=\varepsilon_{1},
\end{gathered}
$$

so that $L\left(\omega_{1}^{4}+i \omega_{2}^{4}, \omega_{1}^{7}-i \omega_{2}^{7}\right)=-\varepsilon_{1} u_{6}$.

Using similar methods we consecutively obtain that

$$
\begin{aligned}
& L\left(\omega_{1}^{2}+i \omega_{2}^{2}, \omega_{1}^{8}-i \omega_{2}^{8}\right)=-\sqrt{3} \epsilon_{2} \mu i u_{7} \\
& L\left(\omega_{1}^{3}+i \omega_{2}^{3}, \omega_{1}^{8}-i \omega_{2}^{8}\right)=-\sqrt{3} \epsilon \mu i u_{7} .
\end{aligned}
$$

Note that by applying (??) on

$$
h\left(L\left(\omega_{1}^{3}+i \omega_{2}^{3}, \omega_{1}^{8}-i \omega_{2}^{8}\right), L\left(\omega_{1}^{2}+i \omega_{2}^{2}, \omega_{1}^{5}-i \omega_{2}^{5}\right)\right),
$$

we see that $\epsilon=-\epsilon_{2}$. Using similar arguments, we proceed to find that

$$
\begin{aligned}
& L\left(\omega_{1}^{4}+i \omega_{2}^{4}, \omega_{1}^{8}-i \omega_{2}^{8}\right)=\sqrt{3} \epsilon_{1} \epsilon_{2} \mu i u_{5} \\
& \epsilon_{1}=\epsilon_{2} \\
& L\left(\omega_{1}^{5}+i \omega_{2}^{5}, \omega_{1}^{6}-i \omega_{2}^{6}\right)=-\sqrt{3} \mu i u_{2} \\
& L\left(\omega_{1}^{5}+i \omega_{2}^{5}, \omega_{1}^{7}-i \omega_{2}^{7}\right)=-\sqrt{3} \mu i u_{3} \\
& L\left(\omega_{1}^{5}+i \omega_{2}^{5}, \omega_{1}^{8}-i \omega_{2}^{8}\right)=-\sqrt{3} \mu i u_{4} \\
& L\left(\omega_{1}^{6}+i \omega_{2}^{6}, \omega_{1}^{7}-i \omega_{2}^{7}\right)=\sqrt{3} \mu i \epsilon_{2} u_{4}, \\
& L\left(\omega_{1}^{6}+i \omega_{2}^{6}, \omega_{1}^{8}-i \omega_{2}^{8}\right)=-\sqrt{3} \epsilon_{2} \mu i u_{3}, \\
& L\left(\omega_{1}^{7}+i \omega_{2}^{7}, \omega_{1}^{8}-i \omega_{2}^{8}\right)=\sqrt{3} \mu i \epsilon_{2} u_{2} .
\end{aligned}
$$

Moreover it now immediately follows that $\epsilon_{2}=1$.

At last, we will study the solution given by the case when the metric on $\mathcal{U}$ has signature 3. Start with $u_{1}$ defined as in (40), choose $u_{2} \perp u_{1}$ of length -1 and by surjectivity of $L\left(\omega_{1}^{1}+i \omega_{2}^{1},-\right)$ find $\omega_{1}^{2}, \omega_{2}^{2}$ such that $L\left(\omega_{1}^{1}+i \omega_{2}^{1}, \omega_{1}^{2}-i \omega_{2}^{2}\right)=\sqrt{3} \mu i u_{2}$. Similarly, choose $u_{3} \perp u_{1}, u_{2}$ of length -1 and find $L\left(\omega_{1}^{1}+i \omega_{2}^{1}, \omega_{1}^{3}-i \omega_{2}^{3}\right)=\sqrt{3} \mu i u_{3}$. Then, by lemma (20) we can see that the vector $L\left(\omega_{1}^{2}+i \omega_{2}^{2}, \omega_{1}^{3}-i \omega_{2}^{3}\right)$ is imaginary, therefore, it defines a unit vector $u_{4}$, of length -1 , such that $L\left(\omega_{1}^{2}+i \omega_{2}^{2}, \omega_{1}^{3}-i \omega_{2}^{3}\right)=$ $\sqrt{3} \mu i u_{4}$. Moreover, we find the unique vectors $\omega_{1}^{4}$ and $\omega_{2}^{4}$ such that $L\left(\omega_{1}^{1}+i \omega_{2}^{1}, \omega_{1}^{4}-\right.$ $\left.i \omega_{2}^{4}\right)=\sqrt{3} \mu i u_{4}$ and $L\left(\omega_{1}^{4}+i \omega_{2}^{4}, \omega_{1}^{4}-i \omega_{2}^{4}\right)=\sqrt{3} \mu u_{1}$. Further on, we see that $L\left(\omega_{1}^{2}+\right.$ $\left.i \omega_{2}^{2}, \omega_{1}^{4}-i \omega_{2}^{4}\right)$ and $L\left(\omega_{1}^{3}+i \omega_{2}^{3}, \omega_{1}^{4}-i \omega_{2}^{4}\right)$ are orthogonal to $u_{1}, u_{2}, u_{4}$ and $u_{1}, u_{3}, u_{4}$. We compute by property $(23) h\left(L\left(\omega_{1}^{2}+i \omega_{2}^{2}, \omega_{1}^{4}-i \omega_{2}^{4}\right), L\left(\omega_{1}^{1}+i \omega_{2}^{1}, \omega_{1}^{3}-i \omega_{2}^{3}\right)\right)$ and 
$h\left(L\left(\omega_{1}^{3}+i \omega_{2}^{3}, \omega_{1}^{4}-i \omega_{2}^{4}\right), L\left(\omega_{1}^{1}+i \omega_{2}^{1}, \omega_{1}^{2}-i \omega_{2}^{2}\right)\right)$ and, as the metric on $\left\{u_{2}, u_{3}, u_{4}\right\}^{\perp}$ is positive definite, we find

$$
L\left(\omega_{1}^{2}+i \omega_{2}^{2}, \omega_{1}^{4}-i \omega_{2}^{4}\right)=-\sqrt{3} \mu i u_{3} \quad \text { and } \quad L\left(\omega_{1}^{3}+i \omega_{2}^{3}, \omega_{1}^{4}-i \omega_{2}^{4}\right)=\sqrt{3} \mu i u_{2} .
$$

Next, we choose $u_{5} \perp u_{1}, u_{2}, u_{3}, u_{4}$ of length 1 and find $\omega_{1}^{5}, \omega_{2}^{5}$ such that $L\left(\omega_{1}^{1}+\right.$ $\left.i \omega_{2}^{1}, \omega_{1}^{5}-i \omega_{2}^{5}\right)=\sqrt{3} \mu u_{5}$. Then, we notice by property $(23)$ that $L\left(\omega_{1}^{2}+i \omega_{2}^{2}, \omega_{1}^{5}-\right.$ $\left.i \omega_{2}^{5}\right), L\left(\omega_{1}^{3}+i \omega_{2}^{3}, \omega_{1}^{5}-i \omega_{2}^{5}\right)$ and $L\left(\omega_{1}^{4}+i \omega_{2}^{4}, \omega_{1}^{5}-i \omega_{2}^{5}\right)$ are real vectors and satisfy the orthogonality conditions which allow us to pick $u_{6}, u_{7}, u_{8}$ of length 1 , in their directions respectively, and complete $\left\{u_{1}, u_{2}, u_{3}, u_{4}\right\}$ to an orthonormal basis, that is $L\left(\omega_{1}^{2}+i \omega_{2}^{2}, \omega_{1}^{5}-i \omega_{2}^{5}\right)=\sqrt{3} \mu u_{6}, L\left(\omega_{1}^{3}+i \omega_{2}^{3}, \omega_{1}^{5}-i \omega_{2}^{5}\right)=\sqrt{3} \mu u_{7}$ and $L\left(\omega_{1}^{4}+\right.$ $\left.i \omega_{2}^{4}, \omega_{1}^{5}-i \omega_{2}^{5}\right)=\sqrt{3} \mu u_{8}$. Notice that, by lemmas (20) and property (40) we obtain

$$
\begin{array}{ll}
L\left(\omega_{1}^{6}+i \omega_{2}^{6}, \omega_{1}^{6}-i \omega_{2}^{6}\right)=-\sqrt{3} \mu u_{1}, & L\left(\omega_{1}^{1}+i \omega_{2}^{1}, \omega_{1}^{6}-i \omega_{2}^{6}\right)=\sqrt{3} \mu u_{6}, \\
L\left(\omega_{1}^{7}+i \omega_{2}^{7}, \omega_{1}^{7}-i \omega_{2}^{7}\right)=-\sqrt{3} \mu u_{1}, & L\left(\omega_{1}^{1}+i \omega_{2}^{1}, \omega_{1}^{7}-i \omega_{2}^{7}\right)=\sqrt{3} \mu u_{7}, \\
L\left(\omega_{1}^{8}+i \omega_{2}^{8}, \omega_{1}^{8}-i \omega_{2}^{8}\right)=-\sqrt{3} \mu u_{1}, & L\left(\omega_{1}^{1}+i \omega_{2}^{1}, \omega_{1}^{8}-i \omega_{2}^{8}\right)=\sqrt{3} \mu u_{8} .
\end{array}
$$

In the following, we determine $L\left(\omega_{1}^{2}+i \omega_{2}^{2}, \omega_{1}^{6}-i \omega_{2}^{6}\right)=-\sqrt{3} \mu u_{5}$, as it is a real vector of length $3 \mu^{2}$, orthogonal on $u_{1}, u_{2}, u_{3}, u_{4}, u_{6}$, and given that its component in the direction of $u_{5}$ is $-\sqrt{3} \mu$ ( by property (23)). Furthermore, we find $L\left(\omega_{1}^{2}+\right.$ $\left.i \omega_{2}^{2}, \omega_{1}^{7}-i \omega_{2}^{7}\right)=\varepsilon_{1} \sqrt{3} \mu u_{8}$, as it is orthogonal to $L\left(\omega_{1}^{2}+i \omega_{2}^{2}, \omega_{1}^{k}-i \omega_{2}^{k}\right)$ and $L\left(\omega_{1}^{1}+\right.$ $\left.i \omega_{2}^{1}, \omega_{1}^{7}-i \omega_{2}^{7}\right)$, for $k=2, \ldots, 6$ and $\varepsilon_{1}= \pm 1$. Similarly, we determine for $\varepsilon_{j}= \pm 1$, $j=2, \ldots, 8$ the following vectors

$$
\begin{array}{ll}
L\left(\omega_{1}^{2}+i \omega_{2}^{2}, \omega_{1}^{8}-i \omega_{2}^{8}\right)=\varepsilon_{2} \sqrt{3} \mu u_{7}, & L\left(\omega_{1}^{5}+i \omega_{2}^{5}, \omega_{1}^{6}-i \omega_{2}^{6}\right)=-i \sqrt{3} \mu u_{2} \\
L\left(\omega_{1}^{3}+i \omega_{2}^{3}, \omega_{1}^{6}-i \omega_{2}^{6}\right)=\varepsilon_{3} \sqrt{3} \mu u_{8}, & L\left(\omega_{1}^{5}+i \omega_{2}^{5}, \omega_{1}^{7}-i \omega_{2}^{7}\right)=-i \sqrt{3} \mu u_{3}, \\
L\left(\omega_{1}^{3}+i \omega_{2}^{3}, \omega_{1}^{7}-i \omega_{2}^{7}\right)=-\sqrt{3} \mu u_{5}, & L\left(\omega_{1}^{5}+i \omega_{2}^{5}, \omega_{1}^{8}-i \omega_{2}^{8}\right)=\varepsilon_{8} \sqrt{3} \mu u_{4}, \\
L\left(\omega_{1}^{3}+i \omega_{2}^{3}, \omega_{1}^{8}-i \omega_{2}^{8}\right)=\varepsilon_{4} \sqrt{3} \mu u_{6}, & L\left(\omega_{1}^{6}+i \omega_{2}^{6}, \omega_{1}^{7}-i \omega_{2}^{7}\right)=-\varepsilon_{5} i \sqrt{3} \mu u_{4}, \\
L\left(\omega_{1}^{4}+i \omega_{2}^{4}, \omega_{1}^{6}-i \omega_{2}^{6}\right)=\varepsilon_{5} \sqrt{3} \mu u_{7}, & L\left(\omega_{1}^{6}+i \omega_{2}^{6}, \omega_{1}^{8}-i \omega_{2}^{8}\right)=-\varepsilon_{3} i \sqrt{3} \mu u_{3}, \\
L\left(\omega_{1}^{4}+i \omega_{2}^{4}, \omega_{1}^{7}-i \omega_{2}^{7}\right)=\varepsilon_{6} \sqrt{3} \mu u_{6}, & L\left(\omega_{1}^{7}+i \omega_{2}^{7}, \omega_{1}^{8}-i \omega_{2}^{8}\right)=-\varepsilon_{1} i \sqrt{3} \mu u_{2} . \\
L\left(\omega_{1}^{4}+i \omega_{2}^{4}, \omega_{1}^{8}-i \omega_{2}^{8}\right)=\varepsilon_{7} \sqrt{3} \mu u_{5}, &
\end{array}
$$

Then, we can easily find the relations between the coefficients $\varepsilon_{j}$ using property (23): $\varepsilon_{2}=-\varepsilon_{1}, \varepsilon_{4}=-\varepsilon_{3}, \varepsilon_{6}=-\varepsilon_{5}$ and $\varepsilon_{7}=-1, \varepsilon_{8}=-i$. Moreover, we can find $\varepsilon_{1}=-1, \varepsilon_{3}=1$ and $\varepsilon_{5}=-1$ by applying property (23) successively to $L\left(\omega_{1}^{6}+i \omega_{2}^{6}, \omega_{1}^{7}-i \omega_{2}^{7}\right)$ and $L\left(\omega_{1}^{2}+i \omega_{2}^{2}, \omega_{1}^{3}-i \omega_{2}^{3}\right), L\left(\omega_{1}^{2}+i \omega_{2}^{2}, \omega_{1}^{7}-i \omega_{2}^{7}\right)$ and $L\left(\omega_{1}^{5}+\right.$ $\left.i \omega_{2}^{5}, \omega_{1}^{4}-i \omega_{2}^{4}\right)$, and finally, to $L\left(\omega_{1}^{3}+i \omega_{2}^{3}, \omega_{1}^{8}-i \omega_{2}^{8}\right)$ and $L\left(\omega_{1}^{2}+i \omega_{2}^{2}, \omega_{1}^{5}-i \omega_{2}^{5}\right)$.

\subsection{Two canonical examples.}

When the indefinite signature on $U$ is 7 , we have the following example. Let $\mathfrak{h}_{3}(\mathbb{O})$ denote the set of Hermitian matrices with entries in $\mathbb{O}$, the space of octonions endowed with the Jordan multiplication $\circ$ :

$$
\begin{array}{r}
\mathfrak{h}(\mathbb{O})_{3}=\left\{N \in \mathcal{M}_{3}(\mathbb{O}) \mid \bar{N}^{T}=N\right\}, \\
X \circ Y=\frac{1}{2}(X Y+Y X) .
\end{array}
$$

By definition, we have that the determinant of $N \in \mathfrak{h}_{3}(\mathbb{O})$ is given by

$$
\operatorname{det} N=\frac{1}{3} \operatorname{Tr}(N \circ N \circ N)-\frac{1}{2} \operatorname{Tr}(N \circ N)+\frac{1}{6}(\operatorname{Tr} N)^{3} .
$$

Remark that a matrix $N \in \mathfrak{h}_{3}(\mathbb{O})$ is of the form $N=\left(\begin{array}{lll}\xi_{1} & x_{3} & \overline{x_{2}} \\ \overline{x_{3}} & \xi_{2} & x_{1} \\ x_{2} & \overline{x_{1}} & \xi_{3}\end{array}\right)$, where $\xi_{i} \in \mathbb{R}, x_{i} \in \mathbb{O}$. For more details for the space of octonions see [1]. Next, we define 
$G=\left\{N \in \mathfrak{h}(\mathbb{O})_{3} \mid \operatorname{det}(N)=1\right\}$. We define an action of $G$ on $M_{1}=\left\{\bar{N} A N^{T} \mid N \in G\right\}$ by

$$
\begin{aligned}
& \rho: G \times M_{1} \longrightarrow M_{1} \\
& \rho(N) X=\bar{N} X N^{T},
\end{aligned}
$$

where $A=\left(\begin{array}{ccc}1 & 0 & 0 \\ 0 & -1 & 0 \\ 0 & 0 & -1\end{array}\right)$. By construction, this action is transitive and therefore $M_{1}$ is congruent with $G / H$, where $H=\left\{N \in G \mid A \bar{N} A N^{T}=I\right\}$. Note that $\rho(N)$ can be seen as a linear transformation acting on $\mathbb{R}^{27}$ and a straightforward computation shows that $\rho(N) \in S L(27, \mathbb{R})$. Therefore, $M_{1}$ is an homogeneous affine hypersphere in $\mathbb{R}^{27}$. It is now sufficient to work around a point. We introduce local coordinates around a point $p \in M_{1}$ by taking $y_{1}, \cdots, y_{26}$ such that $\xi 1=1$,

$$
\xi_{2}=y_{1}, \quad \xi_{3}=y_{2}, \quad x_{1}=\sum_{i=0}^{7} y_{3+i} e_{i}, \quad x_{2}=\sum_{i=0}^{7} y_{11+i} e_{i}, \quad x_{3}=\sum_{i=0}^{7} y_{19+i} e_{i},
$$

for $\left\{e_{0}, \cdots, e_{7}\right\}$ a basis of $\mathbb{O}$. Therefore, the parametrization for our hypersurface is given by

$$
\left\{\begin{array}{l}
F: \mathbb{R}^{26} \rightarrow \mathbb{R}^{27} \\
p \longmapsto g(p)^{-\frac{1}{3}}(1, p),
\end{array}\right.
$$

where $p=\left(y_{1}, \cdots, y_{26}\right)$ and $g(p):=\operatorname{det} N$. By using the multiplication table for octonions, we can determine $g(p)$ and then, straightforward computations around the point $N=\left(\begin{array}{ccc}1 & 0 & 0 \\ 0 & -1 & 0 \\ 0 & 0 & -1\end{array}\right)$ allow us to find that the isotropy condition holds for $\lambda=\frac{1}{2}$. Thus, the signature of the metric on $M$ is 16 .

When the indefinite signature on $U$ is 3 , we have the following example.

Consider the set of Hermitian matrices with entries in the split-octonions space endowed with the Jordan multiplication $\circ$, as previously defined. For $\{1, i, j, k, l i, l j, l k\}$ an orthogonal basis of the split-octonion space, the length of a vector $x=x_{0}+$ $x_{1} i+x_{2} j+x_{3} k+x_{4} l+x_{5} l i+x_{6} l j+x_{7} l k$ is given by

$$
h(x, x)=\bar{x} x=\left(x_{0}^{2}+x_{1}^{2}+x_{2}^{2}+x_{3}^{2}\right)-\left(x_{4}^{2}+x_{5}^{2}+x_{6}^{2}+x_{7}^{2}\right) .
$$

We define the manifold in a similar way as in the previous example and, by similar arguments, we get that $M$ is an isotropic affine hypersphere of dimension 26 for which the signature of the metric is 12 .

\section{REFERENCES}

[1] J. C. Baez, The octonions, Bull. Amer. Math. Soc., 39(23),145-205 (2001).

[2] O. Birembaux, M. Djoric, Isotropic Affine Spheres, Acta Mathematica Sinica, English Series, Oct., 2012, Vol. 28, No. 10, pp. 1955-1972.

[3] W. Boothby, An Introduction to Differentiable Manifolds and Riemannian Geometry, Elsevier, Singapore, 2007.

[4] J.L. Cabrerizo, M. Fernández, J.S. Gómez, Rigidity of pseudo-iotropic immersions, J. Geom. Phys. 59 (2009) 834-842.

[5] B.-Y. Chen, Complex extensors and Lagrangian submanifolds in indinite complex Euclidean spaces, Bull. Inst. Math. Acad. Sin. (NS) 31 (3) (2013) 151-179.

[6] F. Dillen, L. Vrancken, Hypersurfaces with parallel difference tensor, Japan. J. Math., Vol. 24, No. 1, 1998.

[7] M.P. do Carmo, Riemannian geometry, Birkhäuser, Boston, 1992.

[8] Z. Hu, H. Li, L.Vrancken, Locally strongly convex affine hypersurfaces with parallel cubic form, J. Differential Geom. 87 (2011), no.2, 239-307.

[9] H. Li, L. Vrancken, X. Wang, Minimal Lagrangian isotropic immersions in indefinite complex space forms, Journal of Geometry and Physics, 62 (2012) 707-723. 
[10] M.A. Magid, Shape operator in Einstein hypersurfaces in indefinite space forms, Proc. Amer. Math. Soc. 84 (1982) 237-242.

[11] S.Montiel, F. Urbano, Isotropic totally real submanifolds, Math. Z. 199 (1988) 55-60.

[12] B.O. Neill, Isotropic and Kaehler immersions, Canad. J. Math. 17 (1965) 907-915.

[13] K. Nomizu, T. Sasaki, Affine differential geometry, Cambridge University Press, 1994.

[14] A.Z. Petrov, Einstein spaces, Pergamon Press, Hungary, Oxford and New York, 1969.

[15] D.B. Shapiro, Compositions of Quadratic Forms, 2000. 24 x 17 cm. 417 pages, Series: de Gruyter Expositions in Mathematics 33.

Marilena Moruz: LAMAV, Université de Valenciennes, Campus du Mont Houy, 59313 VAlenciennes Cedex 9, France

E-mail address: marilena.moruz@gmail.com

Luc Vrancken: LAMAV, Université de Valenciennes, Campus du Mont Houy, 59313 Valenciennes Cedex 9, France; KU leuven, Departement Wiskunde Celestijnenlaan 200B, 3001 Leuven, Belgium

E-mail address: Luc.Vrancken@univ-valenciennes.fr 